\title{
THE EGGLESTON METHOD OF ADMINISTERING DIGITALIS
}

\author{
WITH SOME NOTES ON DIGITALIS LUTEA * \\ S. MARX WHITE, M.D., AND R. EDWIN MORRIS, M.D. \\ MINNEAPOLIS
}

On account of the clinical value of digitalis, the uncertainties in the results and untoward effects after administration have long been the subject of study, especially with a view toward their control or elimination. The attempts to secure derivatives, particularly of definite and uniform activity, are too numerous to discuss here. The fact that the tincture and the infusion are still more widely used than other preparations shows that the derivatives have not satisfied the demand of physicians. Our attempt has been to study these two preparations in such a manner as to control results of therapy by graphic methods, to learn so far as possible the promptitude with which effects can be secured, and the character of such effects.

All digitalis preparations used by us have been standardized according to a modification of the cat method of Hatcher. In relation to digitalis dosage Eggleston' defines the "cat unit" of Hatcher as that amount of the drug calculated per kilogram of cat which is just sufficient to kill when slowly and continuously injected into the vein. This is expressed in terms of milligrams of the drug, whether it be in the pure principle or leaf. The method employed by one of us (Morris) ${ }^{2}$ in standardizing the digitalis preparations is a modification of the Hatcher method similar to that used by Rowntree and Macht. ${ }^{3}$ Various species of Minnesota digitalis, grown by the Department of Pharmacy of the University of Minnesota, were used.

The species lutea attracted particular attention, as the first year leaf, grown in Minnesota, ranks in potency with the higher grades of Digitalis purpurea. In testing Digitalis lutea on cats, Morris noticed the lack of irritation and the quiet lethal period, and suggested the use of Digitalis lutea in man with the hope of finding a preparation causing less than the usual so-called "gastro-intestinal" irritation. As a result

* Submitted for publication Jan. 2, 1918.

* From the Department of Medicine, University of Minnesota.

* Read before the Section on Pharmacology and Therapeutics at the SixtyEight Annual Session of the American Medical Association, New York, June, 1917.

1. Eggleston: Digitalis Dosage, The Archives Int. Med., 1915, 16, 1.

2. Morris: Journal-Lancet, $1917,36$.

3. Rowntree and Macht: Jour. Am. Med. Assn., 1916, 66, 870. 
of this suggestion we have studied the action both of the infusion and tincture of Digitalis lutea clinically in the wards of the University Hospital. The official tincture and infusion of Digitalis purpurea and the corresponding preparations from first-year leaves of Digitalis lutea, both prepared by the Department of Pharmacy, University of Minnesota, have been used. The drugs have been given by one of three methods.

1. The method proposed by Eggleston. The strength of the preparation to be used is determined in "cat units" and from this the dose for man is calculated. The average amount of tincture administered orally required to produce full therapeutic effect (or minor toxic action) and given as above outlined, by the Eggleston method, is about 0.146 c.c. per pound of patient's body weight. (Example: Patient weighs 130 pounds; this multiplied by 0.146 equals 18.98 c.c. Since the infusion is correspondingly weaker in strength, for the infusion give six and two-thirds times this quantity or 126.5 c.c.) The amount thus determined on is given in four doses within a period of eighteen hours by the following formula: The first dose is one-half the total amount to be given; the second, given six hours later, is one half the remainder; and the third and fourth doses, given at six hour intervals, are one half the second dose in amount.

2. The method of Eggleston is followed immediately, or after one or two days, by what may be called tonic doses of infusion or tincture, given three or four times daily over a period of days or even weeks. These doses are usually 0.6 to 1 c.c of the tincture, and 4 to 8 c.c. of the infusion.

3. The tincture or infusion is given in these so-called tonic doses without the previous administration of the larger Eggleston doses.

The results of the Eggleston method with or without the later administration of digitalis are of such a character as to warrant report. In our studies attention is centered on the records obtained with the polygraph and electrocardiograph, correlated with records and notes of clinical observation.

The value of these methods of study should be outlined: The polygraph when used in conjunction with the electrocardiograph, as in this study, is principally of value in giving a record of rate and rhythm of arterial and venous pulsations. The character of waves recorded by this method is of less value, but even here, since changes in the amplitude, sometimes striking in character, are seen, these changes in amplitude have some value since all records here shown are taken by the same observer with the same instrument. ${ }^{4}$

4. Morris' Modifications of the MacKenzie Ink Polygraph, Jour. Am. Med. Assn., 1916, 66, 1922. 
The electrocardiograph gives a record with certain features to which particular attention is paid at this time. Changes in rate and rhythm are noted as with the polygraph. Conduction time, designated $\mathrm{P}-\mathrm{R}$ (or when a $\mathrm{Q}$-wave is present $\mathrm{P}-\mathrm{Q}$ ) interval, is measured principally in records taken from right arm to left leg (D II).

Changes in conductivity in the junctional tissues (bundle of $\mathrm{His}$ and its branches) may occur. In cases with auricular fibrillation it is desirable to depress conductivity, this in all probability being the cause of the slowed ventricular rate and remarkable effects during effective digitalis therapy.

In the normal nonfibrillating case, slowing by digitalis is due to vagus influences which depress stimulus formation at the sinus node. In such cases digitalis may also depress conductivity in the junctional tissues, through both a direct and a vagus action, and this may be shown in two ways:

1. A prolongation of conduction time (P-R or $P-Q$ interval). This prolongation may be considerable, as much as 0.25 second, probably even more, before actual blocking of impulses is likely to occur. When a prolongation of conduction time beyond 0.2 second is found before administering digitalis, the drug must be used very cautiously, if at all, and then only in the presence of very distinct indications. When a normal conduction time (less than 0.2 second) before giving digitalis, gives way to a prolongation after digitalis, the same caution should be observed. In both instances the electrocardiogram provides the best if not the only effective means of study and control.

2. Partial or complete block. (Auriculoventricular disassociation.) If depression in conductivity with increase in conduction time becomes too great, slowing because of missed beats, or of an idioventricular rhythm, occurs. This cannot aid cardiac action, and should be rigidly guarded against wherever possible. The well known effect of atropin in removing a digitalis block is evanescent and requires too much of the drug to be a comfortable safeguard.

A still further effect of digitalis on the heart is the production of extrasystoles through increase of irritability in the myocardium, particularly of the ventricles. When this occurs the well known paired beats may be seen, each pair due to a contraction of the normal supraventricular type, followed by a ventricular extrasystole, and this by a compensatory pause. Extrasystoles often occur before digitalis is given, but when so frequent as to give paired beats, a digitalis influence is probable, and when pairing occurs in a case of auricular fibrillation, is certain.

It has been established by many observers that in the electrocardiogram the normal ventricular complex $(Q, R, S$, and $T$ waves ) remains 
constant in outline in any healthy person. This is true also for individuals with diseased hearts in whom the condition of the heart remains unchanged. Unless alterations occur in the heart, the ventricular complex for any individual remains unchanged through life. So true is this that where repeated tracings from one individual have been taken, identification may often be made from an electrocardiogram.

Comparison of the electrocardiograms taken from the same individual when free from digitalis effects and when under the influence of digitalis may show changes in conduction time and in outline of the complexes, as well as change in the rate and rhythm. Lewis ${ }^{5}$ says:

The duration of the normal P-R interval as measured in Lead II may vary from 0.13 to 0.21 second. It usually lies between 0.13 and 0.16 second. A P-R interval of 0.16 second and accompanying heart rates of 90 and over is probably of pathologic duration. A P-R interval of 0.20 second and over is probably of pathologic duration in all but exceptional instances, whatever the heart rate may be which it accompanies.

Referring to the T-wave, Lewis ${ }^{5}$ says:

$\mathrm{T}$ is always an upward variation in Lead II. It may show partial inversion in Lead I on rare occasions; partial or complete inversion in Lead III is relatively common, and occurs especially in association with splintering of the curves during the opening phases of ventricular systole.

The most valuable and extensive contribution on the modifications in the electrocardiogram produced by digitalis is by Cohn, Fraser and Jamieson. ${ }^{\circ}$ They assert that in curves of patients under treatment with digitalis, alterations in the $T$ waves of the electrocardiograms occurred in a large number (thirty times in thirty-four patients), and that the change was detected before alteration in rhythm or conduction time had occurred and. before symptoms referable to the gastro-intestinal tract disturbed the patients (except in five instances). Other changes in the ventricular complex were noted. They say that an alteration in the curve may be taken as evidence that the influence of digitalis is being exerted on the heart, and that changes in the $\mathrm{T}$-waves were detected after an equivalent of $1.2 \mathrm{gm}$. or even less of the dried leaves of digitalis were given; that is to say, on the third day of administration; on several occasions they show the altered forms after thirtysix to forty-eight hours.

Clinical observation comprises a study of features familiar to all. Eggleston $^{1}$ describes the principal points in clinical improvement as slowing in pulse and respiration rates, the more or less rapid subsidence of dyspnea, orthopnea, and persistent cough, and clearing of lung bases; in fibrillation, decrease in pulse deficit, the clearing of congested liver and splanchnic region with loss of pulsation and tenderness, and

5. Lewis: Philosoph. Trans., Royal Soc., London, Series B, 1912, 202.

6. Cohn, Fraser and Jamieson: Jour. Exper. Med., 1915, 21, 593. 
diminution in size; the diminution in cyanosis, improvement of cold extremities, the disappearance of edema and increase in urinary output.

In cases of auricular fibrillation records are shown of simultaneous apical and radial rates, illustrating the so-called pulse deficit and the changes in the rate and deficit under digitalis.
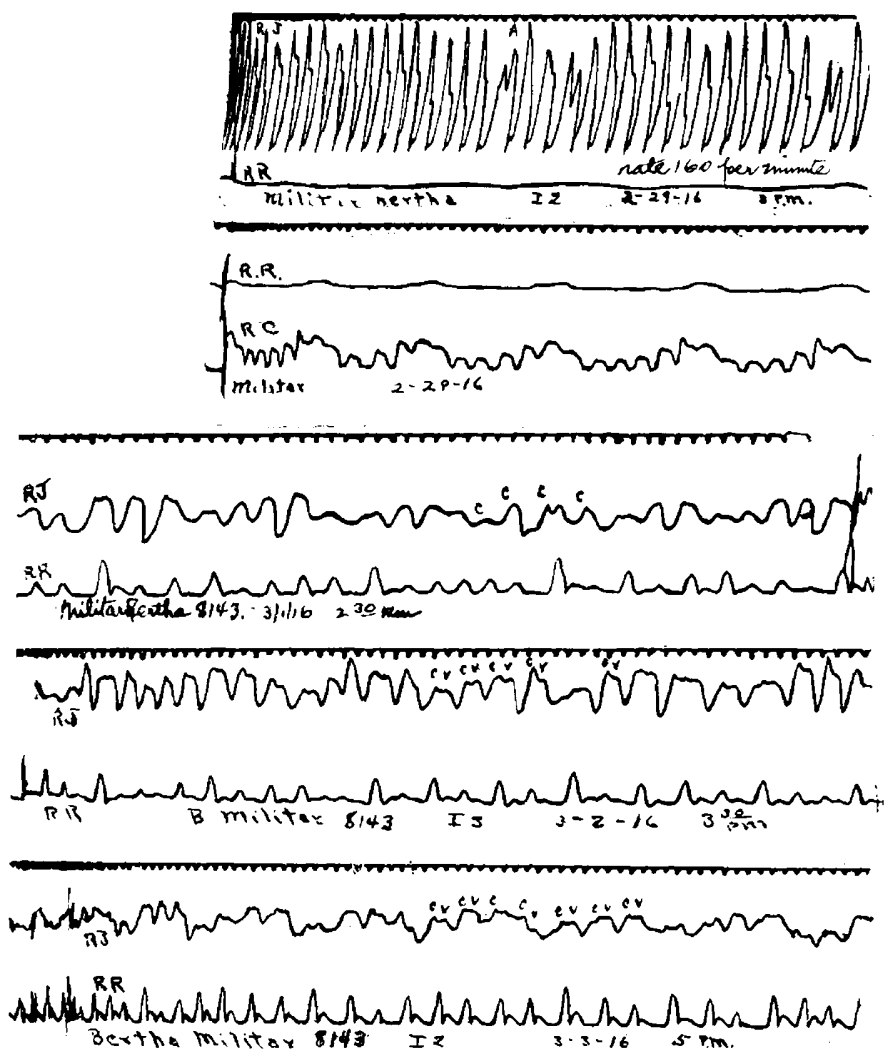

Fig. 1.-(Case 1.) Polygrams, Feb. 29, 1916, from right radial ( $R$ R) right jugular $(\mathrm{R} \mathrm{J})$ and right Carotid $(\mathrm{R} \mathrm{C})$. Radial pulse almost imperceptible but shows expiratory filling and expiratory collapse. Carotid shows large, and jugular, enormous, waves, total arrhythmia. March 1, March 2 and March 3, 1916, show successive increase in radial, and decrease in jugular pulse waves, with distinct effect within twenty-four hours.

\section{REPORT OF CASES}

CAse 1.-B. M., woman, aged 32 (Hosp. No. 8143), was admitted Feb. 28, 1916.

Clinical diagnoses: (1) chronic valvular disease of heart and mitral regurgitation; (2) chronic myocardial degeneration with decompensation; (3) auricular fibrillation; (4) passive congestion of viscera; (5) ascites and anasarca.

Weight not determined on admission; a very small woman estimated to weigh about 120 pounds, with tremendous amounts of fluid in tissues and peritoneum.

Digitalis was given as follows:

Feb. 29, 1916. Infusion digitalis 45 c.c. 
March 1, 1916. Infusion digitalis 15 c.c. (total 60 c.c. in cighteen hours; onehalf Eggleston dosage given because of fluid).

March 2, 1916. Infusion digitalis 75 c.c.

March 3, 1916. Infusion digitalis 15 c.c. (total 90 c.c. in eighteen hours; three-fourths Eggleston dosage, following half Eggleston dosage of preceding two days).

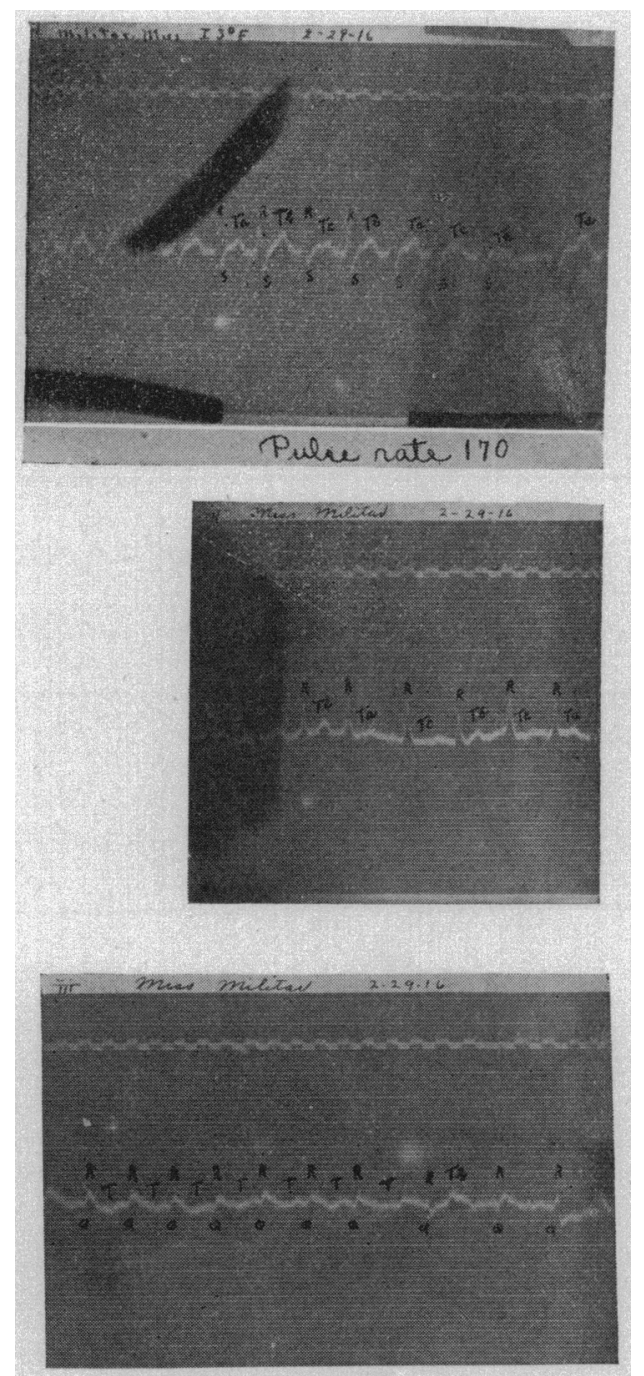

Fig. 2.-(Case 1.) Feb. 29, 1916, auricular fibrillation, pulse rate 170 , total arrhythmia. T-wave positive in D I and D II, slightly inverted in D III, but varies in outline probably because of the deflections of galvanometer string due to the auricular fibrillation.

Then 45 c.c. infusion were given daily except for short periods following toxic symptoms.

Polygrams Feb. 29, 1916; March 1, 1916; March 2, 1916, and March 3, 1916 (Fig. 1). show total arrhythmia (auricular fibrillation) and marked improvement in radial pulse, with lessening of excessive pulsation in jugulars and carotids. 
Feb. 29, 1916. Electrocardiogram (Fig. 2) (before digitalis was given). $T$ positive in D I and D II, inverted in D III. Pulse rate average 170. Auricular fibrillation.

March 1, 1916. Electrocardiogram (Fig. 3). T positive in D I and inverted in D II and III. Pulse rate 155. (General condition considerably improved.)

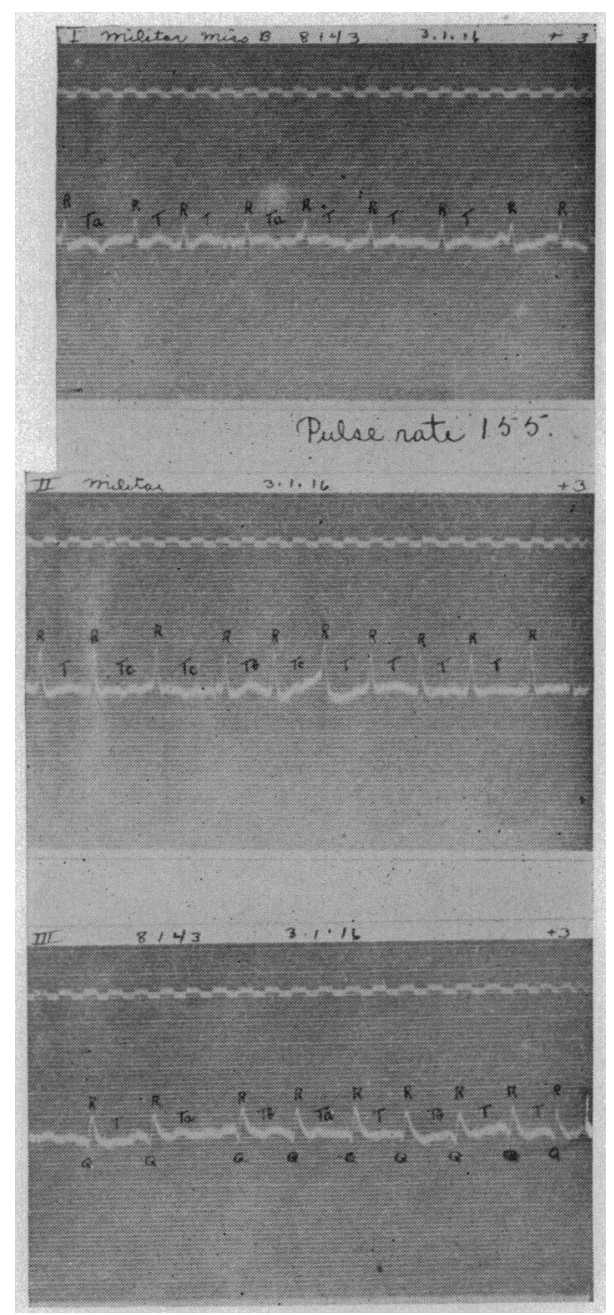

Fig. 3.-(Case 1.) March 1, 1916, auricular fibrillation continues, pulse rate 155. T-wave positive in D I and inverted in D II and D III.

March 2, 1916. Electrocardiogram (Fig. 4). Tracings similar to March 1, 1916. Pulse rate average, 130. (Patient shows marked improvement clinically.) March 8, 1916. Electrocardiogram (Fig. 5). T shows distinct inversion in D I, D II and D III. Pulse rate average 90.

In this case, one of auricular fibrillation with alarming decompensation, the digitalis effect was very apparent in less than twenty-four hours, and within forty-eight hours after beginning the drug, the 
patient was feeling so much better that she wanted to get up and about. The change in the $T$-wave is only moderate in degree and develops rather slowly, although it is apparent within twenty-four hours after beginning digitalis therapy. The reduction in pulse rate, probably through blocking the less effective impulses through the bundle of His, is striking.

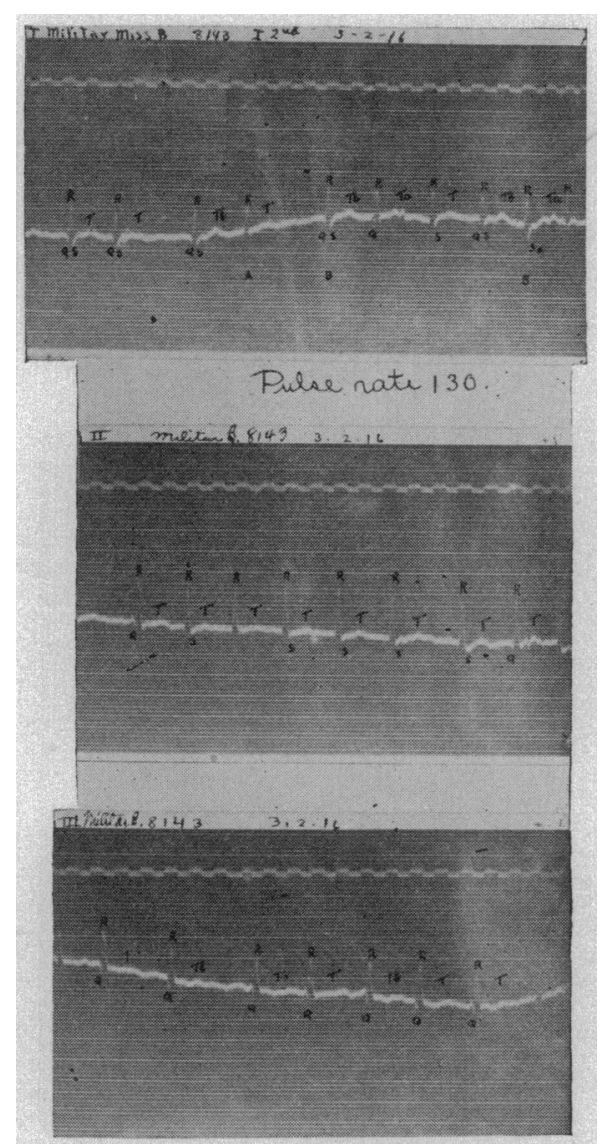

Fig. 4.-(Case 1.) March 2, 1916, tracing shows pulse irregular, rate averaging 130, with $\mathrm{T}$-wave showing little if any change from previous day.

An intercurrent erysipelas of the thigh developed March 12, subsiding within ten days.

Later, after prolonged rest and digitalis therapy, the patient developed regular pulse with $\mathrm{P}$-waves shown in electrocardiograms. This occurred after at least six months of auricular fibrillation. Later, the patient developed pericarditis and died Oct. 14, 1916, seven and onehalf months after this study. 
Necropsy diagnoses: 1. Adranced chronic and acute serofibrino hemorrhagic pericarditis. 2. Acute myocarditis (myomalacia cordis). 3. Dilatation of heart. 4. Chronic passive congestion of liver, spleen and kidneys. 5. Mild acute parenchymatous nephritis.

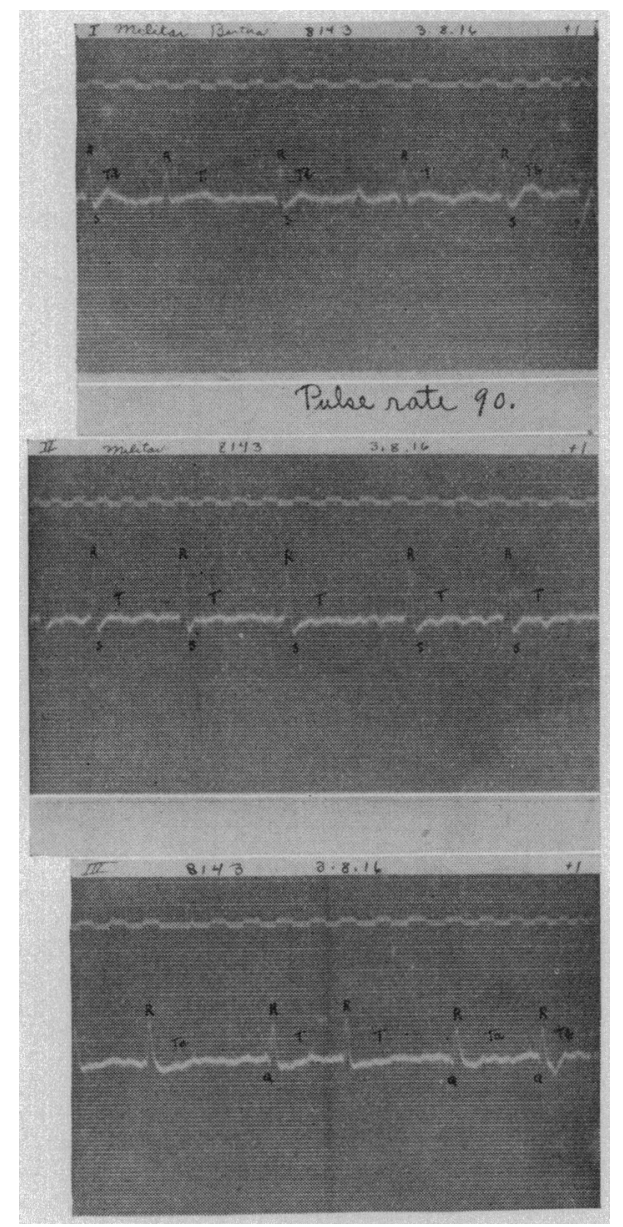

Fig. 5--(Case 1.) March 8, 1917, marked slowing, rate 90, irregular, with the small deflections and auricular fibrillation. T-wave inverted in D I, D II and D III.

CASE 2.-F. F., man, aged 30 (Hosp. No. 8669).

Clinical diagnoses: (1) mitral and aortic insufficiency; (2) exophthalmic goiter; (3) auricular fibrillation; (4) chronic amygdalitis; (5) alveolar abscesses; pyorrhea alveolaris.

Digitalis was given as follows:

May 10, 1916. Infusion digitalis 142.5 c.c. ( $4 \frac{3}{4}$ ounces).

May 11,1916 . Infusion digitalis 52.5 c.c. ( $1 \frac{3 / 4}{4}$ ounces).

(The foregoing constituting for this patient the calculated "Eggleston dosage.") 
May 15, 1916. Infusion digitalis 187.5 c.c. (61/4 ounces) ; "Eggleston dosage."

May 31, 1916. Infusion digitalis 60 c.c. ( 2 ounces).

June 1,1916 . Infusion digitalis 60 c.c. (2 ounces).

May 10, 1916, and May 11, 1916 (Fig. 6), four polygrams showing marked improvement in rate and character of pulse; May 10 shows rate 163 per minute; May 11 shows rate 98 per minute; total arrhythmia, due to auricular fibrillation.

May 14, 1916; May 15, 1916, and May 16, 1916 (Fig. 7), five polygrams show total arrhythmia. The two lower tracings, which are continuous, show onset and offset of a period of "paired" beats which developed next day, May 16, 1916, after the second Eggleston dose.

May 17, 1916. Electrocardiogram (Fig. 8). Auricular fibrillation. $\mathrm{T}$ isoelectric in D I, negative $1 \mathrm{~mm}$. in D II. Paired beats, each pair consisting of a normal supraventricular type of complex followed by a ventricular extrasystole.
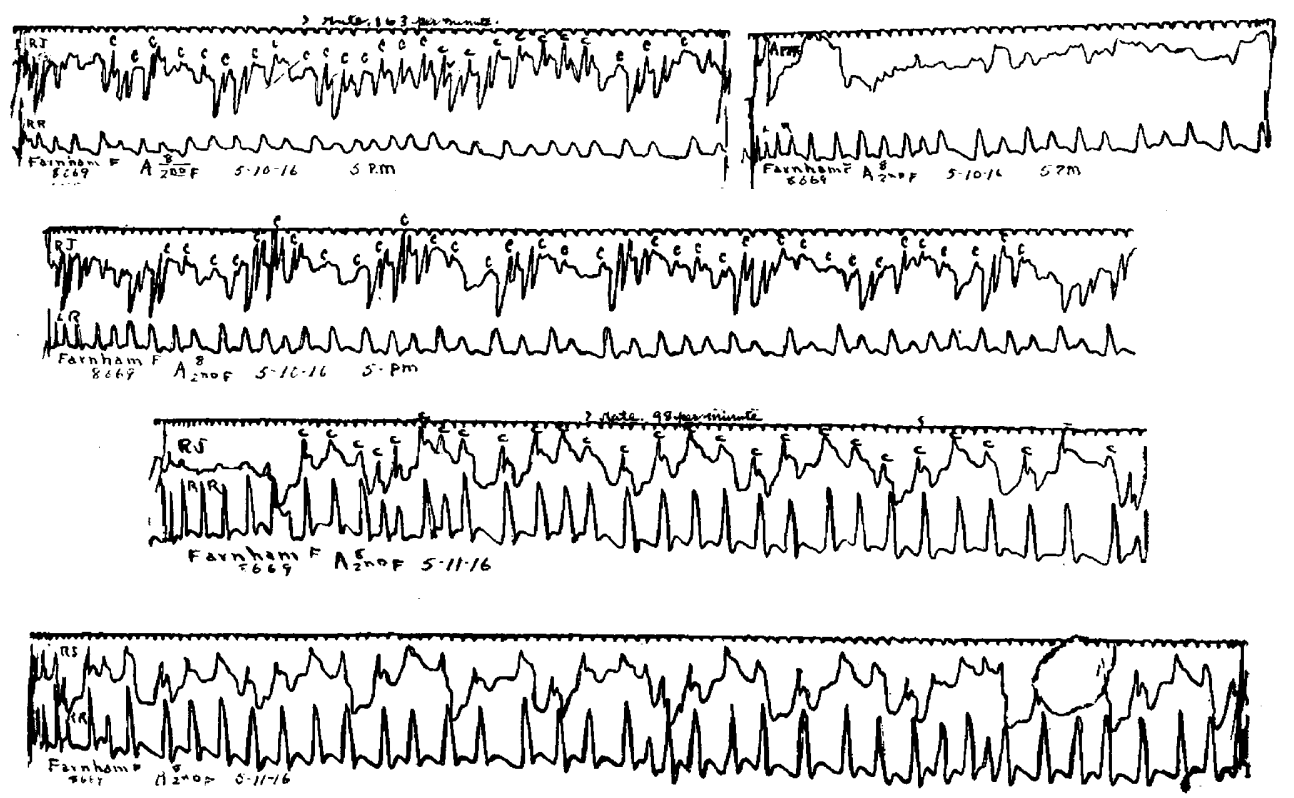

Fig. 6.-(Case 2.) Polygrams, May 10, 1916, right jugular (R J) and right radial ( $R$ R), apex and left radial ( $L R)$, and right jugular ( $R J)$ and left radial ( $L$ ) show small radial and large jugular pulsations. Rate 163; total arrhythmia, due to auricular fibrillation. May 11, 1916, shows marked lessening of jugular and increase in radial pulse wave, with development of pulsus celer type of wave. Total arrhythmia; rate 98.

June 21, 1916. Electrocardiogram (Fig. 9). (No digitalis for twenty days.) Auricular fibrillation. Rate increased to 120 per minute. $T$ positive in D I and D II, slightly negative, then positive in D III.

July 12, 1916. Electrocardiogram (Fig. 10) (no digitalis for forty-one days). Auricular fibrillation; ventricular rate 153 per minute; $T$ a distinct positive wave in D I, D II and D III.

In this patient, the pulse rate showed a deficit, between apical and radial, varying from 8 beats to 36 per minute. May 15, after the second Eggleston dosage, the apical rate, which had been between 118 and 140, dropped to 104 within twelve hours and remained at or below 
this point for thirty-six hours longer, gradually rising again to a maximum of 154 on May 19. Marked clinical improvement with increase of urinary output while on a constant diet and fluid intake accompanied the slowing of rate, as shown by totals of twenty-four hour urines as follows :

May 12, 250 c.c.; May 13, 250 c.c.; May 14, 300 c.c.; May 15, 550 c.c.; May 16, 425 c.c.; May 17, 400 c.c.; May 18, 325 c.c.; May 19, 200 c.c.
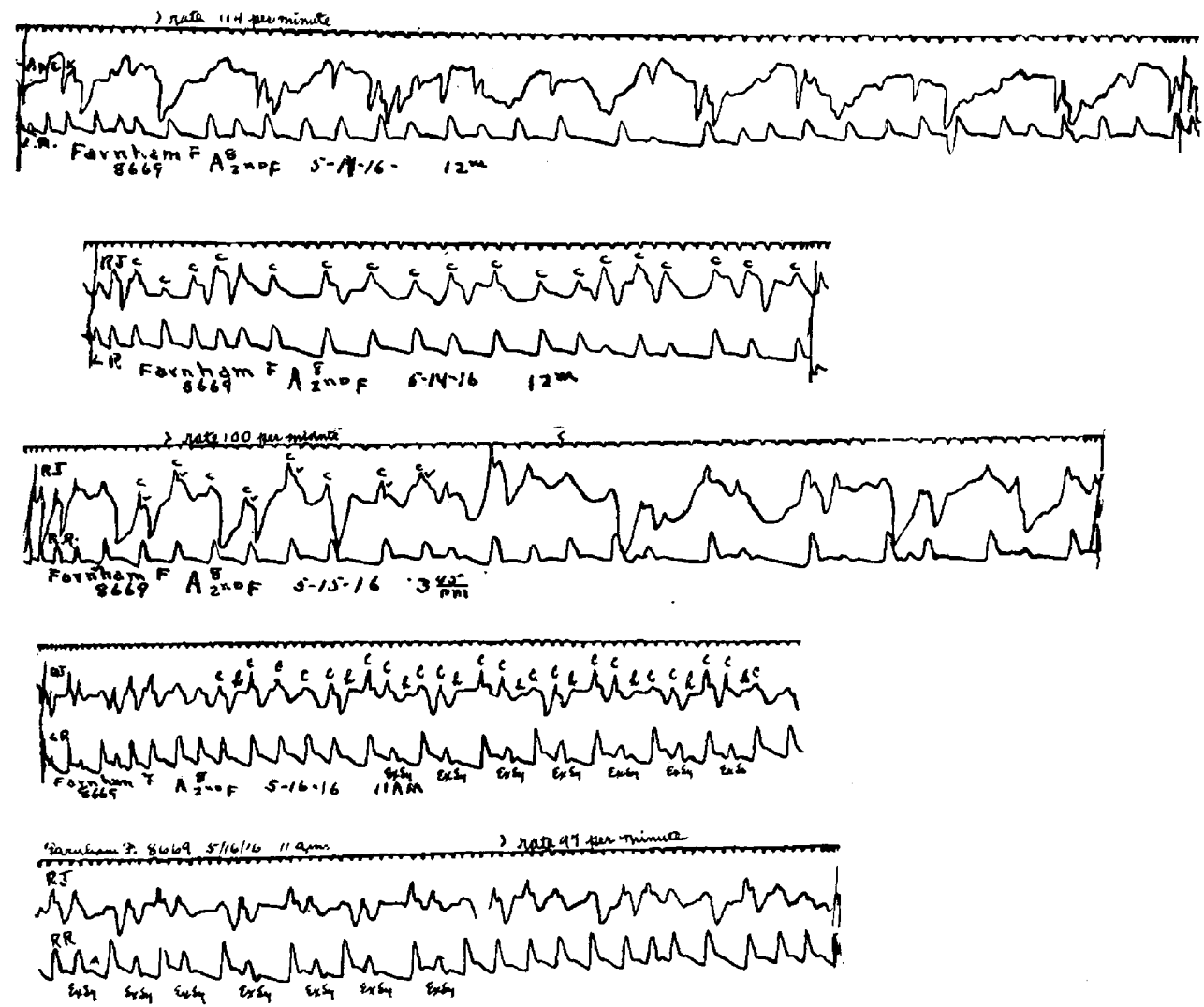

Fig. 7.-(Case 2.) Polygrams, May 14, 1916, show total arrhythmia; rate 114. (Apex and left radial, and right jugular and left radial.) May 15, 1916, shows same arrhythmia, rate 100 per minute. May 16, 1916, the two lower tracings, which are continuous, show the onset and offset of a short period of paired beats, every second (smaller) beat an extrasystole. The fundamental arrhythmia is apparent even during this period.

There is not only marked and prompt improvement in this case, with the reduction in pulse rate and changes in T-wave within twentyfour hours, but after the second Eggleston dose, following the first by only four days, the appearance of extrasystoles shows the irritant action of digitalis on the ventricles, indicating overdosage. This lasted 
five days, then disappeared. Fortunately there seemed to be no interference with the patient's clinical improvement. A digitalis effect is shown twenty days after the last dose.

The patient refused to take even ordinary precautions after leaving hospital and died suddenly at home about three months later.

Case 3.--Mrs. J. P., aged 48 (Hosp. No. 10034), was admitted Nov. 17, 1916; discharged Jan. 17, 1917.

Clinical diagnoses: (1) chronic myocarditis with auricular fibrillation; (2) aortic and mitral insufficiency; (3) edema of lungs; (4) anasarca; (5) chronic amygdalitis.

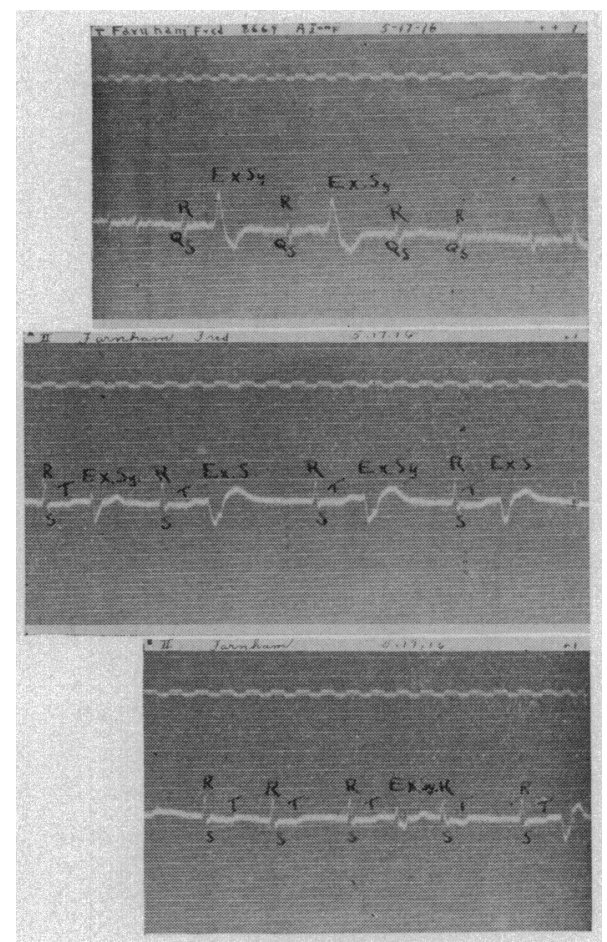

Fig. 8.-(Case 2.) Auricular fibrillation. $\mathrm{T}$ is iso-electric in D I, inverted in D II (two lower strips). Paired beats, due to regularly recurring ventricular extrasystoles.

Weight, 135 pounds. The patient was given infusion Digitalis lutea Nov. 18, 1916, and Nov. 19, 1916, 130 c.c., "Eggleston dosage."

Nov. 18, 1916. Electrocardiogram (Fig. 11). Auricular fibrillation. T shows slight inversion in D I; slight inversion and then positive in D II; slightly positive in D III; pulse rate 96.

Nov. 20, 1916. Electrocardiogram (Fig 12). Auricular fibrillation. Inversion $\mathrm{T}$-wave in D I and D II; pulse rate 70 .

Nov. 21, 1916. Electrocardiogram (Fig. 13). Auricular fibrillation. T-wave shows still greater inversion; pairing of beats, shown only in D III; pulse rate 55 .

Nov. 24, 1916. Pulse rate 55, and by Dec. 19, 1916, the pulse rate was back to 95 , at which time the electrocardiographic tracing shows ventricular complex free from digitalis influence, with $\mathrm{T}$ upright. 
Within the twenty-four hours following the first dose of Digitalis lutea, a tremendous output of urine began, 2,700 c.c. (900 c.c. intake) with 1,800 c.c. output ( 600 c.c. intake) the next day, the output remaining more than the intake for eight days, while after this time

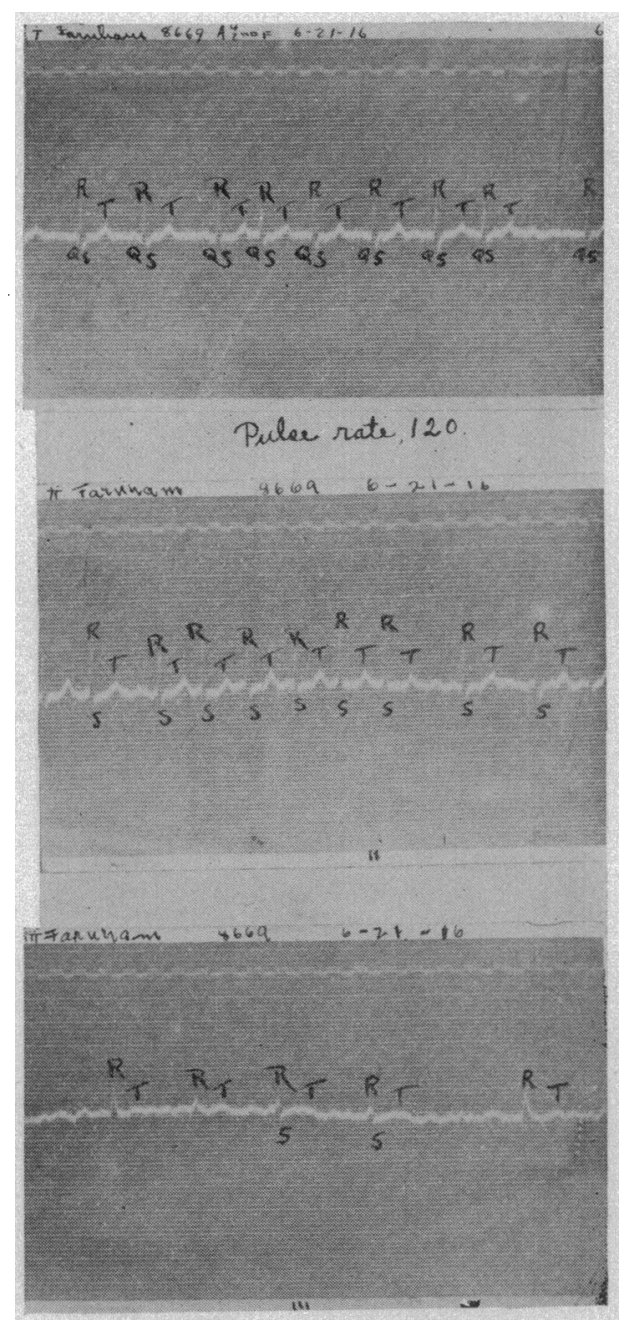

Fig. 9.-(Case 2.) (No digitalis for fourteen days) shows fibrillation of auricles, with increase in rate of ventricles, and disappearance of paired beats, with change in T-wave toward the digitalis-free type. (See Fig. 10.)

the intake constantly exceeded output. Clinical improvement was marked, and began within twenty-four hours after beginning the drug. That this patient had had some digitalis before entering the hospital cannot be excluded. It is suggested by the rate 96 , and by moderate 
inversion of $\mathrm{T}$-wave before beginning the drug. It was not enough, however, and distinct improvement followed effective administration. Increased irritability of ventricle, as shown by paired beats (D III, Fig. 13), showed not until nearly forty-eight hours after suspending the drug. There was no apparent ill effect from it.

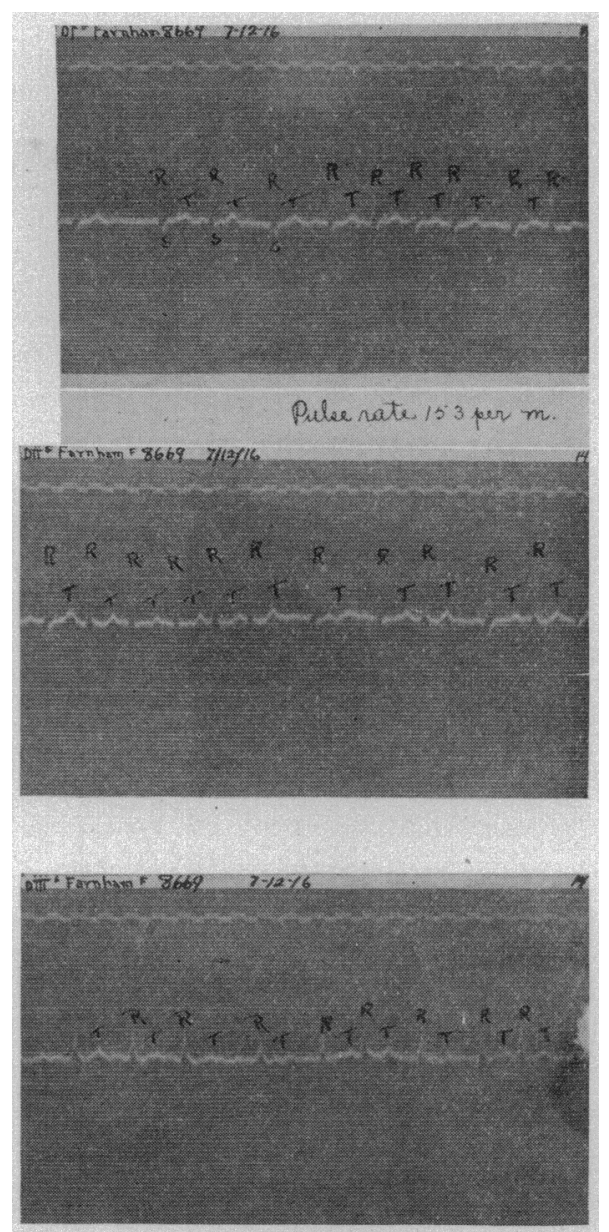

Fig. 10--(Case 2.) (No digitalis for forty-one days.) Ventricular rate has increased again almost to the figures before digitalis was used. (Compare first tracing in Fig. 6 polygram.) T-wave again distinctly upright, though modified frequently by deflections caused by auricular fibrillation.

Case 4.-Mrs. A. R., aged 39 (Hosp. No. 10881), was admitted March 20, 1917 ; discharged May 22, 1917.

Clinical diagnoses: (1) goiter; (2) chronic valvular disease of heart with mitral insufficiency, and decompensation; (3) auricular fibrillation; (4) passive congestion of viscera; (5) ascites and anasarca; (6) chronic amygdalitis.

Weight 96 pounds. The patient received tincture Digitalis lutea 14 c.c. in eighteen hours ("Eggleston dosage") March 24, 1917, and March 25, 1917. 
The electrocardiogram shows auricular fibrillation with inversion of T-wave and slowing of rate from 130, March 24, 1917, to 80, March $26,1917$.

The chart (Fig. 14) shows reduction of pulse deficit, with marked reduction of rate as taken at the apex, but little change in rate as taken at the radial. The apical rate is permanently below its former level within sixteen hours after the first dose.

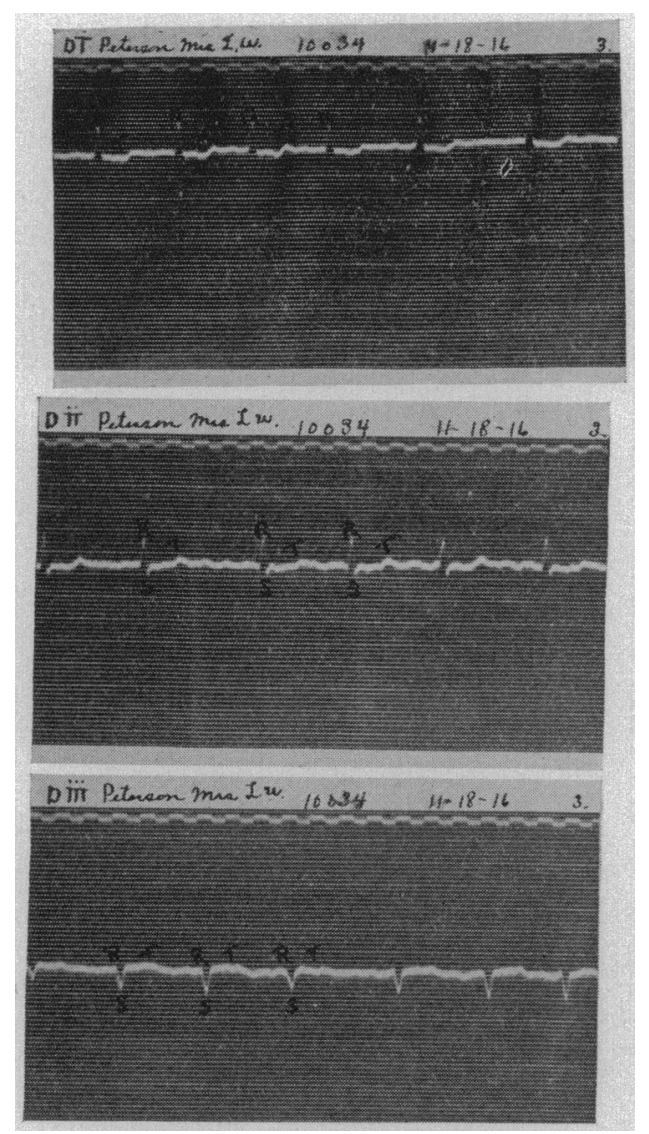

Fig. 11.-(Case 3.) Auricular fibrillation, pulse rate 96, with inversion of first portion of T-wave in D I and D II; suggests some digitalis effect. Note increasing inversion after Eggleston method (see Fig. 12).

A chart (Fig. 15), giving intake and output of fluids with a study of sodium chlorid output in urine and stools while on a diet with known constant salt intake and a daily purgative dose of Epsom salts, shows marked increase in fluid and sodium chlorid output immediately following the drug. We are indebted to Dr. Floyd E. Grave, in charge of the chemical laboratory, Department of Medicine, for this study. The patient showed marked clinical improvement. 
CASe 5.-Mrs. L. M., aged 49 (Hosp. No. 10904), was admitted March 24, 1917; discharged May 19, 1917.

Clinical diagnoses: (1) chronic valvular disease of heart with mitral insufficiency; (2) myocardial degeneration with auricular fibrillation; (3) hypertrophy and dilatation of heart; (4) chronic passive congestion of viscera; (5) infarct of lung; (6) ascites; (7) chronic amygdalitis; (8) dental caries.

The patient received infusion Digitalis lutea, 60 c.c. March 24, 1917 (threefourths Eggleston dosage).

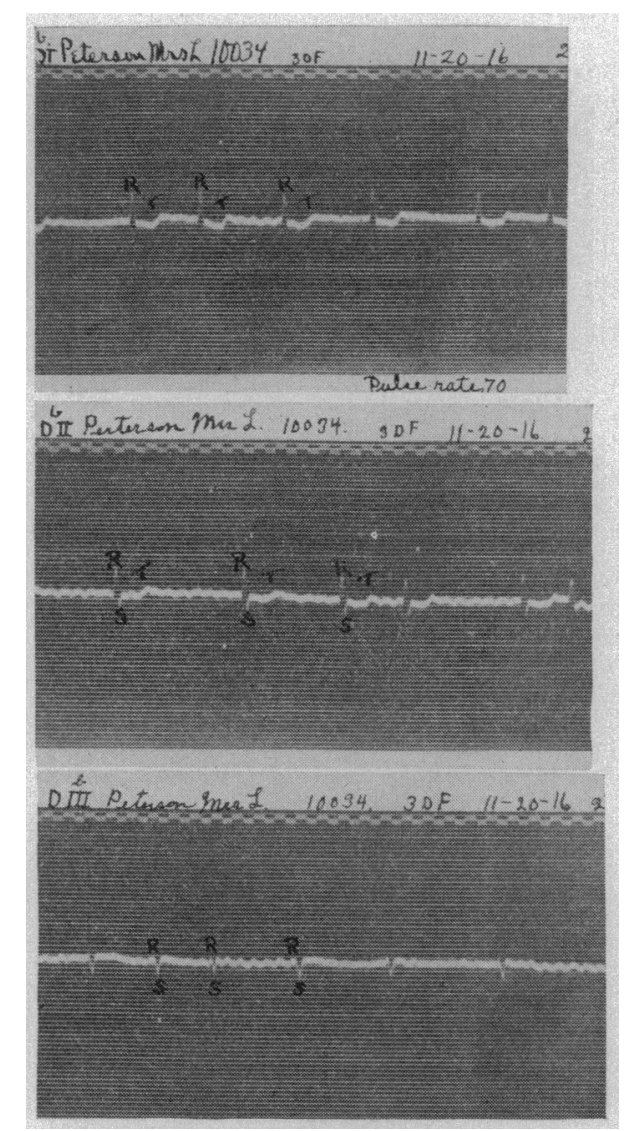

Fig. 12.-(Case 3.) Fibrillation continues, pulse rate 70 , slower within thirty. six hours, and $\mathrm{T}$-wave shows marked inversion in D I and D II.

Electrocardiographic tracings show inversion of $\mathrm{T}$-wave in twentyfour hours after administration of digitalis.

Pulse chart (Fig. 16) shows striking reduction of pulse deficit and slowing of both apical and radial rates within eighteen hours after beginning digitalis. There was some amelioration of symptoms but no decided clinical improvement at first.

Fluid charts could not be made because of a pronounced psychosis. 
Karell diet, complete rest, and digitalis in "tonic" doses, gradtally brought about improvement, and at the time of leaving the hospital no evidences of decompensation could be found.

Case 6.-G. D. M., man, aged 55.

Clinical diagnoses: (1) simple goiter (large); (2) mitral insufficiency (mild grade); (3) myocarditis (moderate grade); (4) paroxysmal auricular flutter.

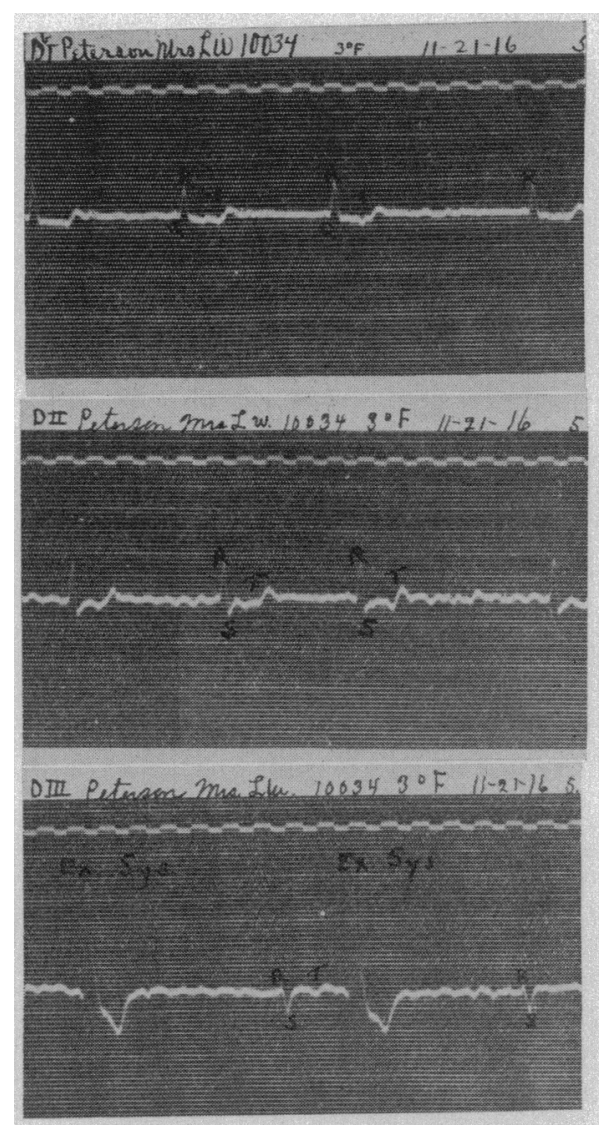

Fig. 13.-(Case 3.) Pulse rate 55. Auricular fibrillation of very coarse type. $\mathrm{T}$ shows marked inversion. In D III frequent ventricular extrasystoles cause paired beats.

For about eight months the patient had occasional attacks of "palpitation" of the heart, coming on usually after dinner in the evening, but occasionally after overexertion. There was no precordial pain. During the attack he became somewhat dyspneic and the attacks lasted from a few minutes to one-half hour.

Digitalis was taken as follows: infusion digitalis, 150 c.c. in twenty-four hours (Eggleston method) beginning Sept. 19, 1916, followed by same infusion 90 c.c. daily until Sept. $23,1916$.

Sept. 14, 1916. Electrocardiogram (had no digitalis). Tracing taken during attack of auricular flutter. Auricular rate 324 per minute; ventricular rate 162 per minute. 


\section{The Intmeristty of Alinuerota}

\section{UNIVERSITY HOSPITALS}

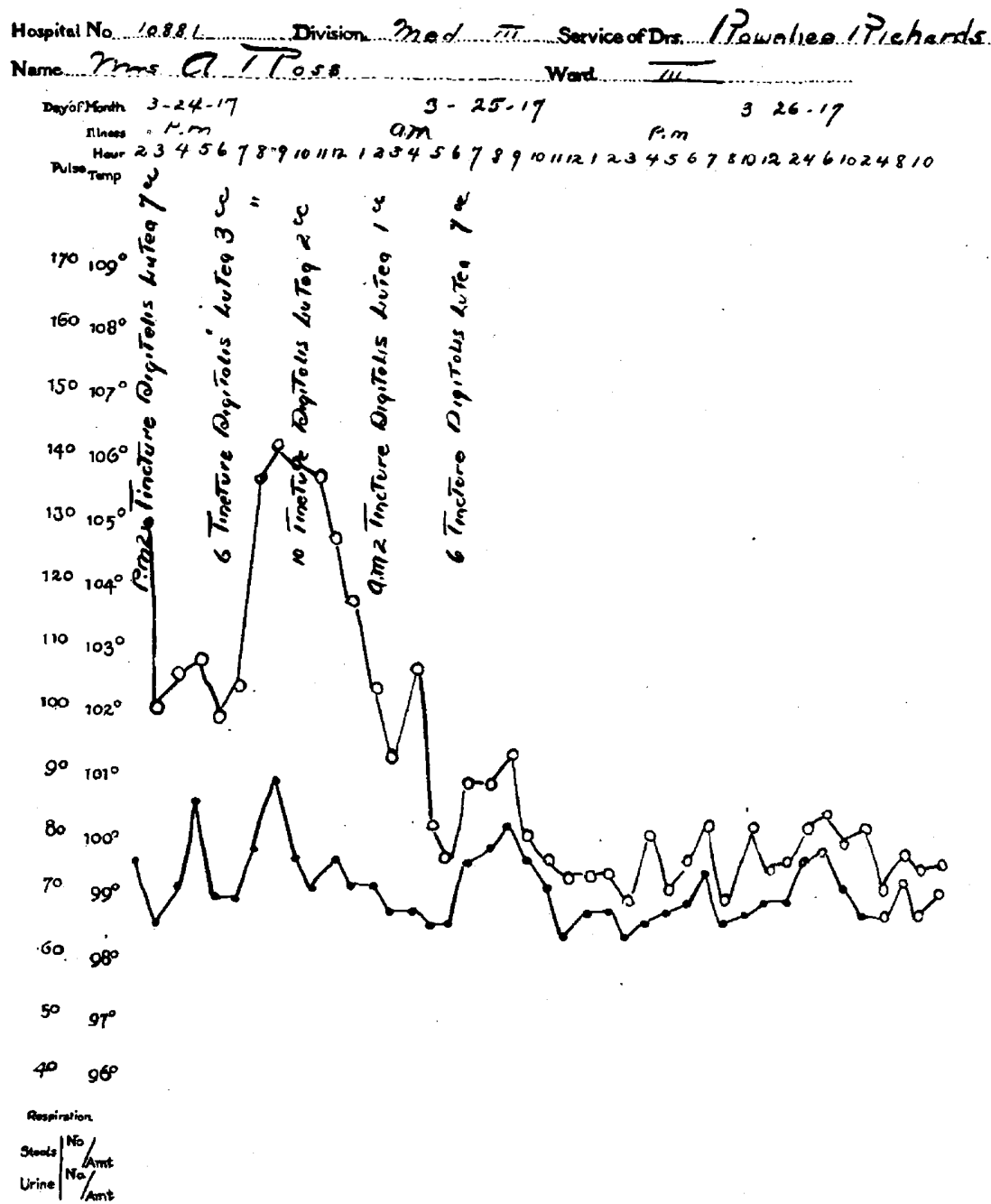

Fig. 14.-(Case 4.) Pulse chart showing rates taken at heart apex (upper lines joining circles) and at radial (lower lines joining dots). The deficit decreases by reduction in apical rate. The radial rate. remains stationary, and if this alone were studied false conclusions regarding digitalis effect on heart rate would be drawn. 
Sept. 19, 1916. Electrocardiogram (before beginning digitalis). P-Q interval 0.15 second in D II. Positive in all derivations.

Sept. 21, 1916. Electrocardiogram (took infusion digitalis 150 c.c. Sept. 19 and 20). P-Q interval 0.16 second in D II. Definite flattening of T-waves in all derivations.

The patient appeared at the clinic Sept. 23, 1916, during a paroxysm, undoubtedly of auricular flutter. The pulse rate was 160 and perfectly regular as felt at the wrist. He was taken to electrocardiographic station, but by the time the apparatus was adjusted the rapid, regular rate had disappeared and been replaced by absolute arrhythmia. A polygram taken at $9: 45 \mathrm{a} . \mathrm{m}$. shows absolute arrhythmia, rate 110 per minute. A polygram taken at $9: 55$ a. $\mathrm{m}$. shows rate 130, perfectly regular. A good jugular tracing could not be obtained. A tracing taken Sept. 25, 1916, two days later, shows regular rhythm, with good jugular waves (normal condition).

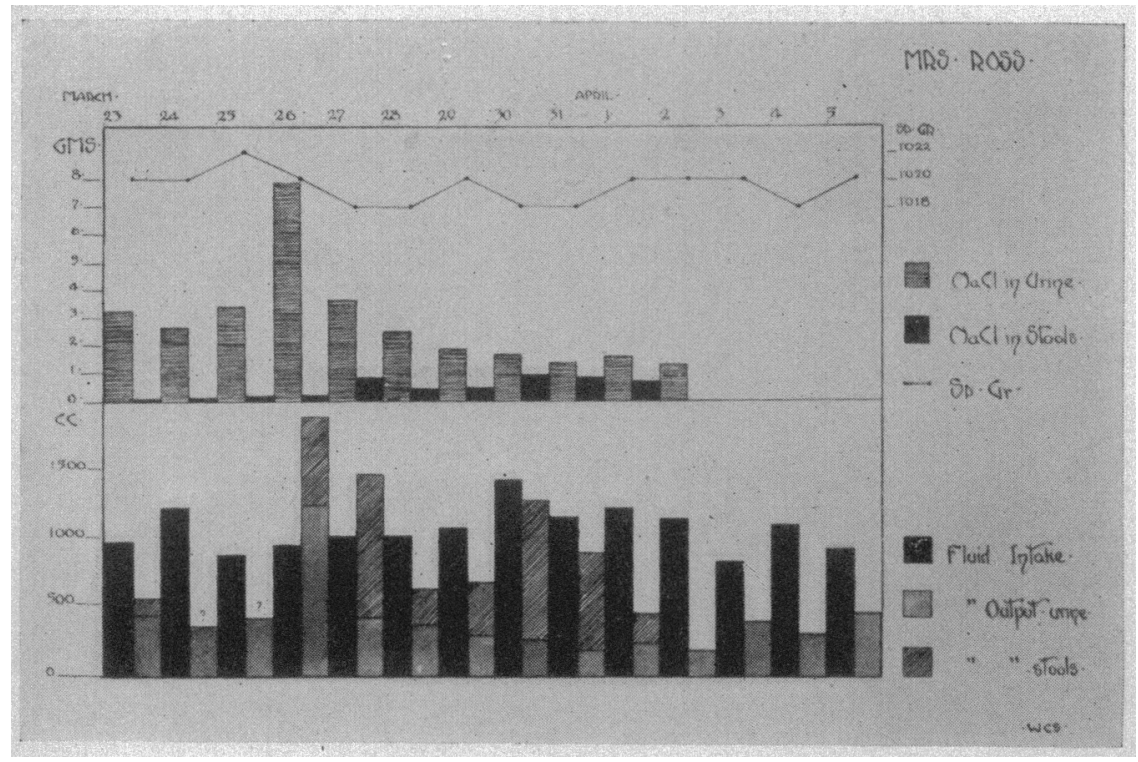

Fig. 15.-(Case 4.) Showing intake of fluids and output of sodium chlorid in urine and stools. Marsh 24 and 25, the amount of fluid in stools was not accurately measured, but there was no radical error as shown by the amounts of sodium chlorid recovered. (Middle chart.)

Sept. 23, 1916. Electrocardiogram, taken at 9:40-41-42 a. m., presenting auricular fibrillation during subsidence of flutter, shows a rate of 110 with absolute arrhythmia. In D I, T is much flattened; T-wave inverted in D II and D III.

Sept. 23, 1916. Electrocardiogram taken at 9:50-51-52 a. m. shows a normal sequence of auricular and ventricular complexes ten minutes after Figure 21 was taken. $T$ is flattened in D I, inverted in D II and D III ; P-Q interval 0.16 second in D II; one extrasystole shown in D I.

Sept. 25, 1916. Electrocardiogram (still taking digitalis in "tonic" doses). $T$ is inverted during first portion, then slightly positive in $D$ I; more marked inversion of $T$ than formerly in D II and D III.

This patient had considerable natusea after ten days on infusion of digitalis. He was later given Digitalis lutea in larger doses than for- 
merly, with no nausea. The use of digitalis lessened the frequency and shortened the duration of the paroxysms. Later, after thyroidectomy, the paroxysms remained absent for six months and the patient

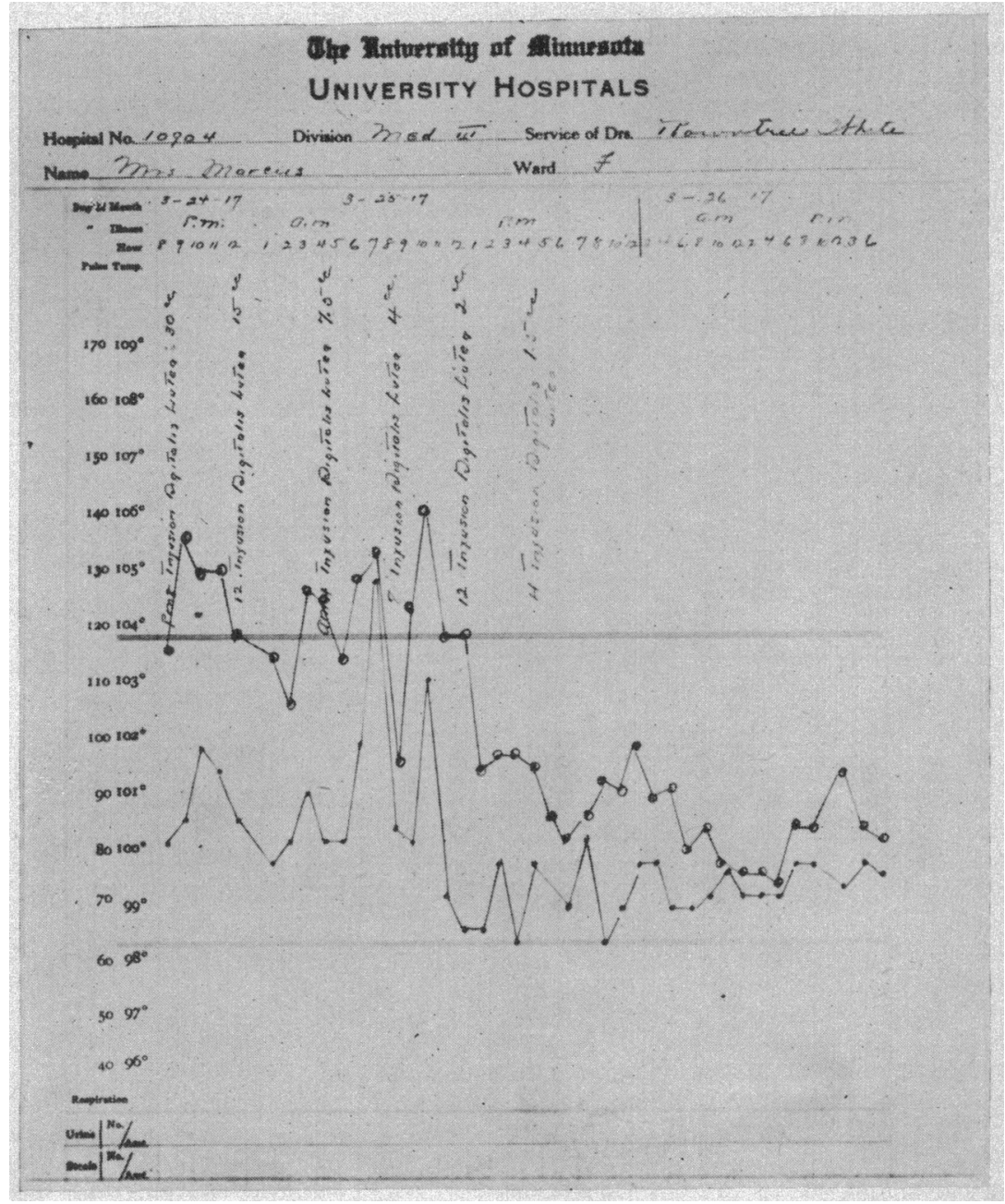

Fig. 16.-(Case 5.) Pulse chart showing decrease in rate and deficit, with prompt digitalis effect in eighteen hours. In this case the deficit, early, sometimes decreased in part by improvement in character of radial pulse.

was able to do full work. This case is reported in greater detail and with tracings in a short paper by us on "Paroxysmal Auricular Flutter."

CASE 7.-John M., man, aged 32 (Hosp. No. 8256), was admitted March 16, 1916; discharged June 15, 1916.

Clinical diagnoses: (1) exophthalmic goiter, a case of moderate severity, with 
enlarged thyroid; (2) nervousness, tremor, weakness, exophthalmos, tachycardia with regular pulse, and a blowing systolic murmur (probably functional) over the mitral area.

Weight 165 pounds. The patient was given infusion digitalis May 2, 1916, 165 c.c. (Eggleston dosage), followed by 90 c.c. May 3.

May 2, 1916. Electrocardiogram before digitalis was given. The pulse rate was 135 per minute while at heart station; when quiet in bed the chart shows 100 to 105 . T positive in D I, D II and D III.

May 5, 1916. Electrocardiogram. T inverted, then slightly positive in D I; inverted and slightly positive in D II; inverted D III.

May 9, 1916. Electrocardiogram. T inverted and positive in D I; inverted, then slightly positive in D II; inverted in D III.

The pulse rate slowed from 100 and 105 before digitalis to 90 and 95 after digitalis and remained so for two weeks, then returned to 105 and 110 per minute, coincident with return of the $T$-wave to its normal upright form. The principal evidence of digitalis effect in this case is in the moderate slowing of pulse rate and in the change in T-wave. This effect is apparent at least six days after withdrawing the drug. No change in conduction time is found.

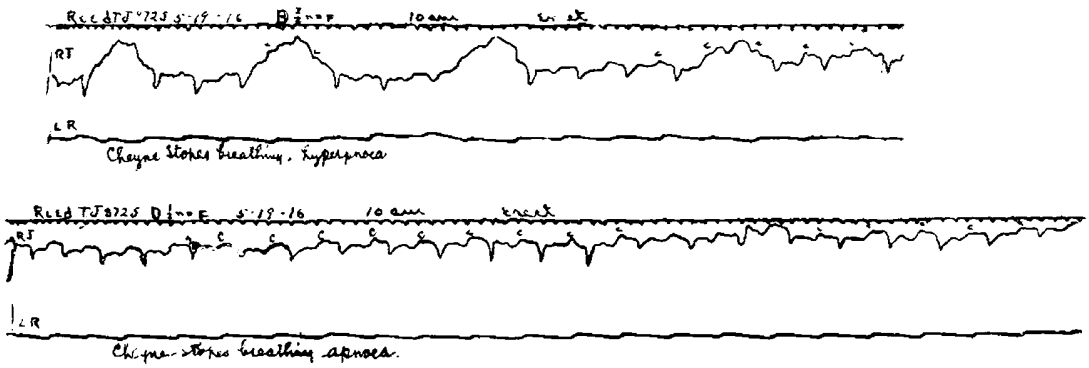

Fig. 17.--(Case 10.) Polygrams May 19, 1916 (before digitalis was given). Impossible to get good radial or jugular tracings; pulse barely perceptible with finger. Shows period of hyperpnea, and of apnea, respectively, in Cheyne-Stokes breathing. (Compare with Fig. 18.)

CASE 8.-Mrs. A. L., aged 26 (Hosp. No. 9861 ).

Clinical diagnoses: (1) retroversion of uterus; (2) enlarged thyroid; (3) pyorrhea alveolaris.

Weight, 110 pounds. The patient received infusion Digitalis lutea, 108 c.c. (Eggleston method) Nov. 5, 1916, then 3 c.c. daily (min. 45) Nov. 7, 1916, to Nov. 27, 1916.

Nov. 4, 1916. Electrocardiogram. T inverted, then $1.5 \mathrm{~mm}$. positive in D I; inverted, then slightly positive in D II; inverted, then slightly positive in D III. Normal P-R interval.

Nov. 10, 1916. Electrocardiogram. T distinctly flattened in D I and D II, inverted in D III.

Nov. 25, 1916. Electrocardiogram. T inverted $1 \mathrm{~mm}$., then slightly positive in D I ; inverted $2.55 \mathrm{~mm}$. in D II ; inverted $1.55 \mathrm{~mm}$. in D III. Normal P-R interval.

Nov. 29, 1916. Electrocardiogram resembles that of Nov. 25, 1916.

The pulse rate before giving digitalis showed a maximum of 120 , minimum of 78 , average for three days, 88 . An intercurrent diarrhea, 
November 6, with severe abdominal pain and temperature 99.4 for a few hours, was accompanied by pulse rate of 96 to 106 ; then the rate gradually dropped to 72 , November 11 , and a minimum of 68 , November 19 , but was always subject to rise on slight provocation.
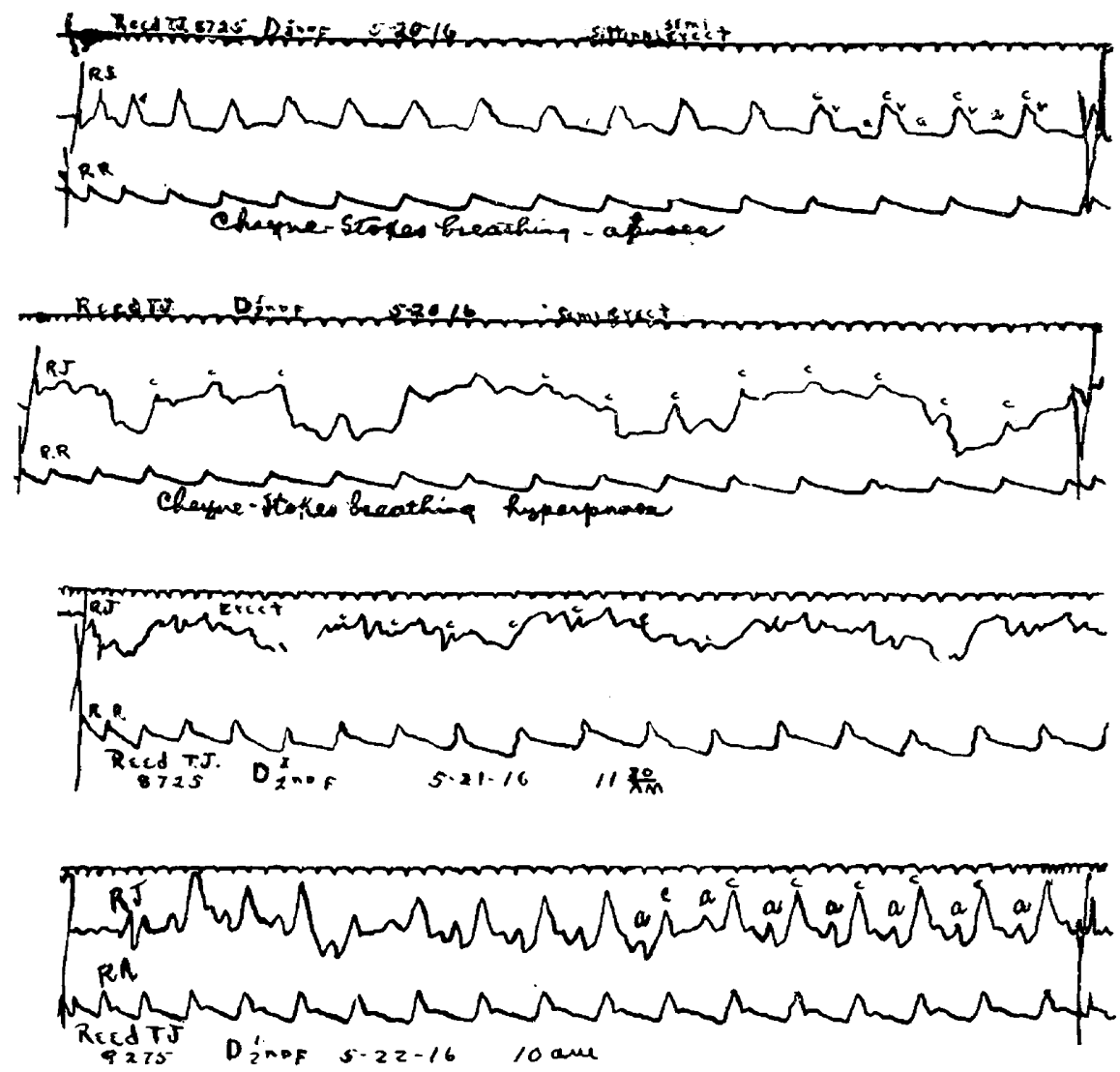

Fig. 18.-(Case 10.) Polygrams May 20, 1916 (compare with Fig. 17), show marked improvement in character of radial pulse (which is regular) within twenty-four hours after beginning digitalis. May 21, 1916, shows greater improvement, and lowest tracing, May 22, 1916, shows auricular waves clearly.

A distinct sinus arrhythmia was noted in this patient. The only indication for digitalis was the rather high rate. The physician referring the case suspected hyperthyroidism on this account, but this was not confirmed by the staff physician. Marked improvement, probably due as much to rest as to drug, occurred.

Case 9.-J. O. (Hosp. No. 10026), was admitted Nov. 16, 1916. Died Nov. 30, 1917.

Clinical diagnoses: (1) carcinoma of stomach with metastatic carcinoma liver; (2) ascites; (3) pleural effusion, left; (4) dental caries. 
Weight 185 pounds. The patient was given infusion Digitalis lutea as follows: Nov. 17, 1916, 90 c.c. and Nov. 18, 1916, 90 c.c. (total 180 c.c. "Eggleston dosage"). Nov. 20 to Nov. 22, inclusive, the patient was given tincture Digitalis lutea, 3 c.c. daily. November 23 to November 28 , inclusive, the patient was given tincture Digitalis lutea, 6 c.c. daily.

Nov. 17, 1916. Electrocardiogram. T shows a small negative wave in all leads. $P-Q=0.17$ second. Pulse rate, average 95 .

Nov. 18, 1916. Electrocardiogram. Slight increase in negativity of $\mathrm{T}$-wave. $\mathrm{P}-\mathrm{Q}=0.17$ second. Pulse rate averages 95 .

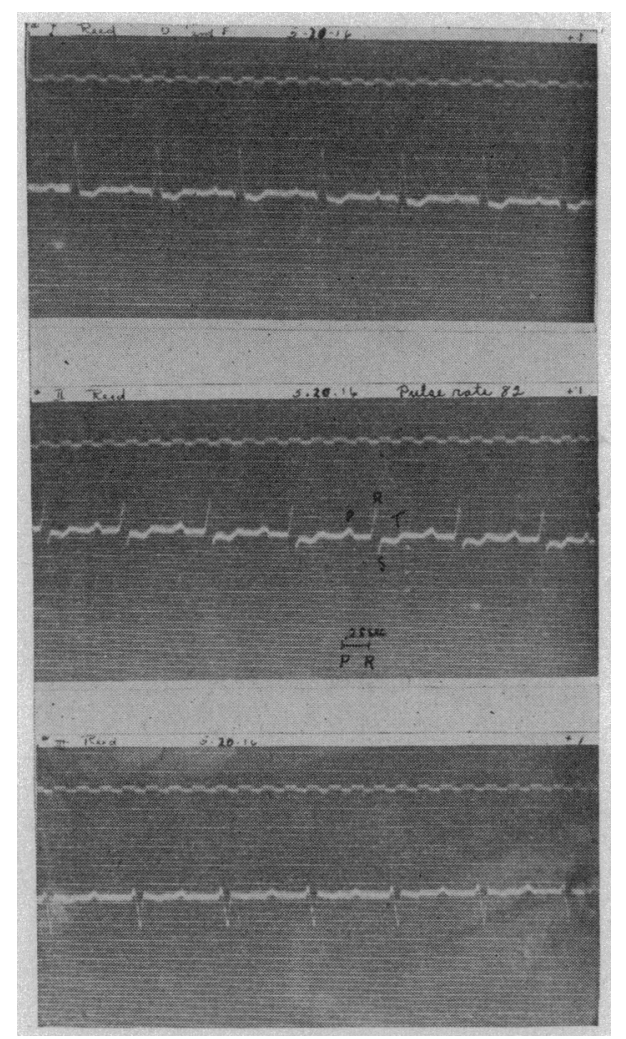

Fig. 19.-(Case 10.) May 20, 1917. P-R $=0.25$ second. T-wave inverted in D I and D II; slight inversion in D III. Pulse rate 82.

Nov. 20 and 21, 1916. Electrocardiogram. T shows greater inversion in D I, November 20; also in D II, Nov. 21, 1916. P-Q=0.17 second. Pulse rate averages 85 .

Nov. 22, 1916. Electrocardiogram. $\mathrm{P}-\mathrm{Q}=0.18$ second. More marked inversion of $T$-wave. Pulse rate averages 72 .

In this case, again, it is possible that some digitalis had been taken before admission, although no history of this could be obtained. An increase in negativity of $\mathrm{T}$-wave is shown within eighteen hours after beginning the drug, and the pulse rate is slowed. No essential change in the conduction time occurred though there is possibly slight slowing November 22, 1916. 
CASE 10.-T. J. R., man (Hosp. No. 8725), was admitted May 18, 1916.

Clinical diagnoses: (1) syphilis; (2) chronic interstitial nephritis; (3) arteriosclerosis (general) ; (4) hypertension (238 systolic, 142 diastolic); (5) myocardial insufficiency.

The blood showed urea nitrogen, 91 ; creatinin, 9.5 ; blood sugar, 0.21 ; phenolsulphonephthalein test, May 20, 1916, 27 per cent. excreted in two hours.

The patient reccived digitalis as follows: May 19, 1916, infusion digitalis, 120 c.c.; May 20, 1916, infusion digitalis, 15 c.c. (total 135 c.c. $=4 \frac{1}{2}$ ounces.

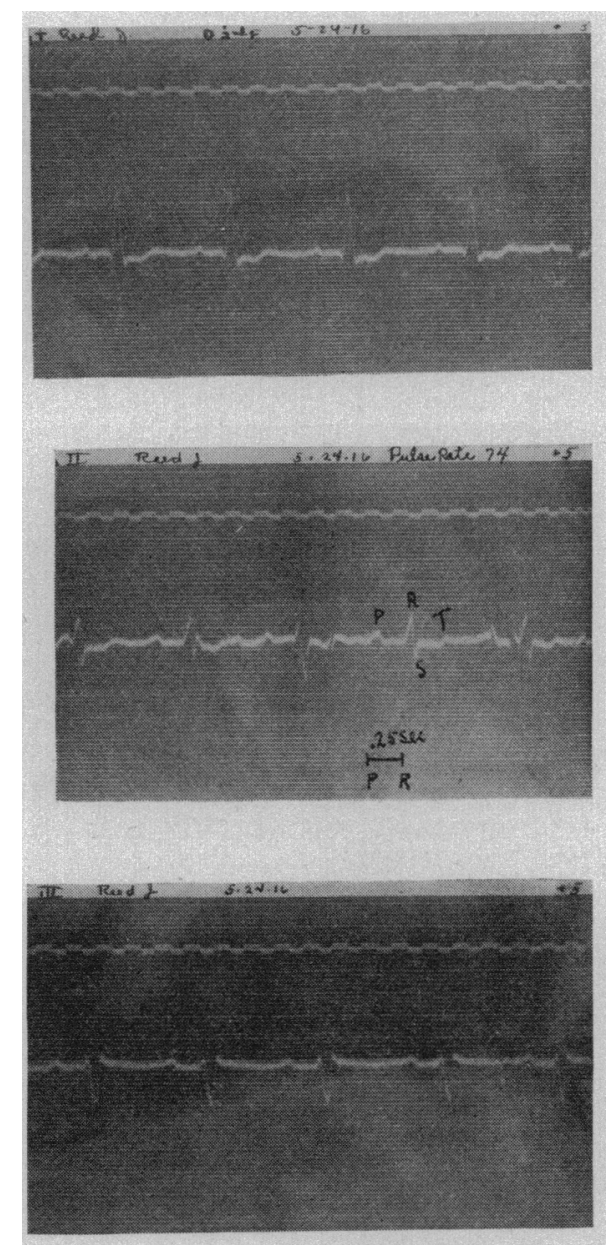

Fig. 20.-(Case 10.) May 24, 1917. $P-R=0.25$ second. Inversion of $\mathrm{T}$-wave increased in all derivations as compared with May 20, 1916 (Fig. 19). Pulse rate 74

"Eggleston dosage"); then none until May 25. May 25 to June 5 the patient received tincture digitalis, minims 30 daily; then none until June 12 , when tincture digitalis 2 c.c. (minims 30) daily were given until June 19 . June 28 , tincture digitalis 8 c.c. (minims 120).

May 19, 1916. Polygram (Fig. 17) (before digitalis was given) shows small pulse; first strip taken during hyperpnea with Cheyne-Stokes breathing; second strip shows period of apnea. 
May 20, 21 and 22, 1916. Polygrams (Fig. 18) show marked improvement in character of pulse wave. Auricular waves have become clearly distinguishable in jugular pulse May 22, 1916.

(No electrocardiographic tracing secured before digitalis was begun, but tracings shown later, July 11, 1916, show less inversion of $\mathrm{T}$-wave than when showing digitalis effects, and $P-R$ interval $=0.21$ second.)

May 20, 1916. Electrocardiogram (Fig. 19). P-R interval $=0.25$ second. $T$ inverted in D I and D II. Slight inversion in D III. Pulse rate 82.

May 24, 1916. Electrocardiogram (Fig. 20). $\mathrm{P}-\mathrm{R}=0.25$ second. $\mathrm{T}$ inverted in D I and D II and slightly inverted in D III. Pulse rate 74.

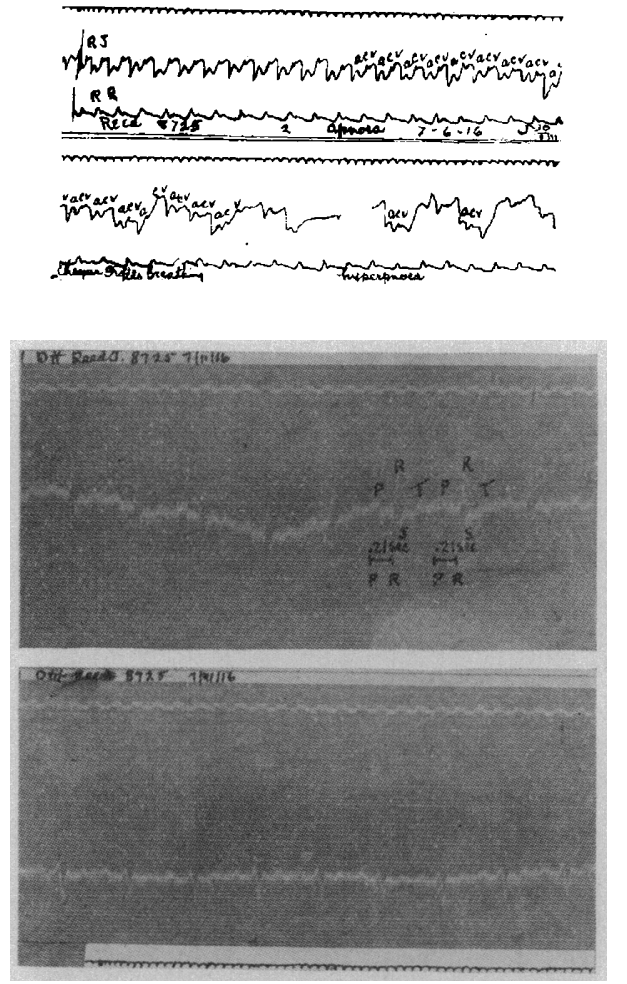

Fig. 21.-(Case 10.) Electrocardiograms July 11, 1916 (no digitalis for thirteen days), P-R $=0.21$ second. T-wave inverted, then positive, in D I (not shown); inverted, then positive in D II and D III (has had no digitalis for thirteen days) (compare with Fig. 19). Two short polygraphic strips.

June 28, 1916. Electrocardiogram. (No digitalis for eight days.) T returning toward normal outline. Pulse rate 65.

July 11, 1916. Electrocardiogram (Fig. 21). No digitalis for thirteen days. $\mathrm{P}-\mathrm{R}$ interval $=0.21$ second. $\mathrm{T}$ inverted, then positive in $\mathrm{D} \mathrm{I}$; inverted and then positive in D II. T inverted, then positive in D III. Polygraphic tracings taken show Cheyne-Stokes breathing in periods of apnea and hyperpnea, respectively.

Died July 12, 1916. Necropsy refused.

It is impossible to say in this case that no digitalis had been taken before admission. It seems probable that there was some digitalis 
effect, although no drugs had been taken for two weeks, since the P-R interval is prolonged to 0.25 second within eighteen hours after beginning the drug, and this interval is shorter at a later time, thirteen days after suspending. We have not seen such a lengthening at such an early date in other instances, except where some digitalis effect was already present. The lengthened $P-R$ interval is the only evidence here of lessened conductivity in the bundle of His. Partial block dio not occur. At one time, May 24, 1916, increased irritability of ventricular muscle is shown by extrasystole.

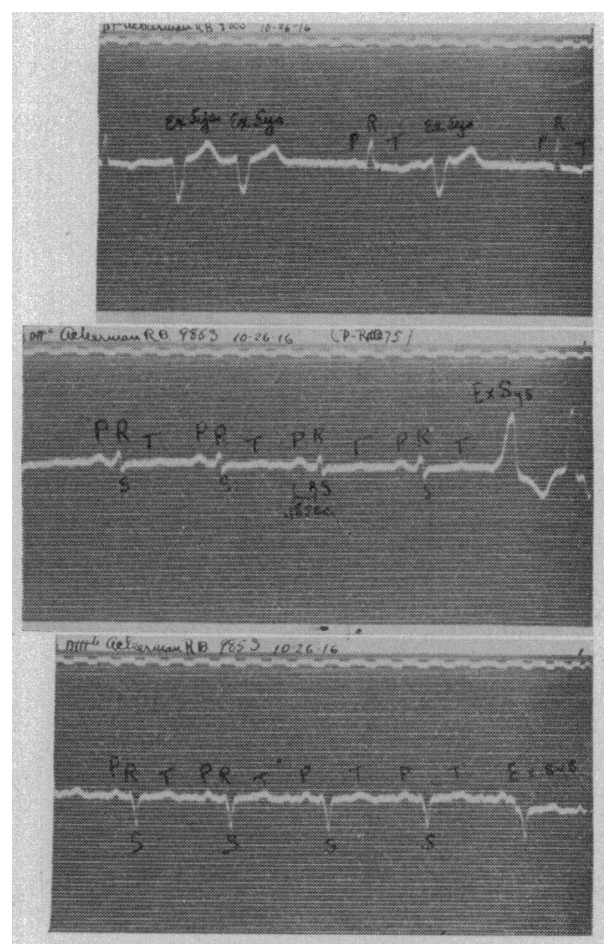

Fig. 22.-(Case 12.) Oct. 26, 1916 (before digitalis was given) $\mathrm{P}-\mathrm{R}=0.18$ second. $T$ slight inversion in $D \mathrm{I}$, iso-electric in early portion, then slightly positive in D II and D III. Extrasystoles frequent. Pulse rate 75.

CASE 11.-Mrs. Sarah M., aged 30 (Hosp. No. 9870), was admitted Oct. 27. 1916; discharged Dec. 21, 1916.

Diagnoses: (1) chronic valvular disease of heart with mitral insufficiency and stenosis; (2) hyperthyroidism (slight); (3) pyorrhea alveolaris and dentat caries; (4) chronic amygdalitis.

Weight, 111 pounds. The patient was given infusion Digitalis lutea as follows:

Nov. 4, 1916, 54 c.c.; Nov. 5, 1916, 54 c.c. (total 108 c.c. "Eggleston doses" in eighteen hours); Nov. 7 to Nov. 17, 1916, tincture digitalis 3 c.c. daily.

Nov. 4, 1916. Electrocardiogram. $\mathrm{P}-\mathrm{R}=0.15$ second. $\mathrm{T} 3 \mathrm{~mm}$. positive in D I $; 1 \mathrm{~mm}$. positive in D II $; 2.5 \mathrm{~mm}$. negative in D III. Pulse rate 80 . 
Now. 9. 1910. Electrocardiogram. Slight inversion of first portion of $T$-wave in $\mathrm{D} I$ and $\mathrm{D}$ II. Inversion of $\mathrm{T}$ wave decreased in $\mathrm{D}$ III. $\mathrm{P}-\mathrm{R}$ interval $=0.17$ second. Prulse rate 92 .

Nov. 17. 1916. Electrocardiogram. $P-R=0.2$ second. T negative, then slight positive in I) 1. Nearly iso-electric and diphasic in D II ; iso-electric and slightly negative in D JII. Pulse rate 88.

Nov. 25. 1916. Electrocardiogram. P-R interval much increased (0.31 second in D II). T shows slight inversion in D $\mathrm{T}$; marked inversion in D II and D IIT. Pulse rate: (x).

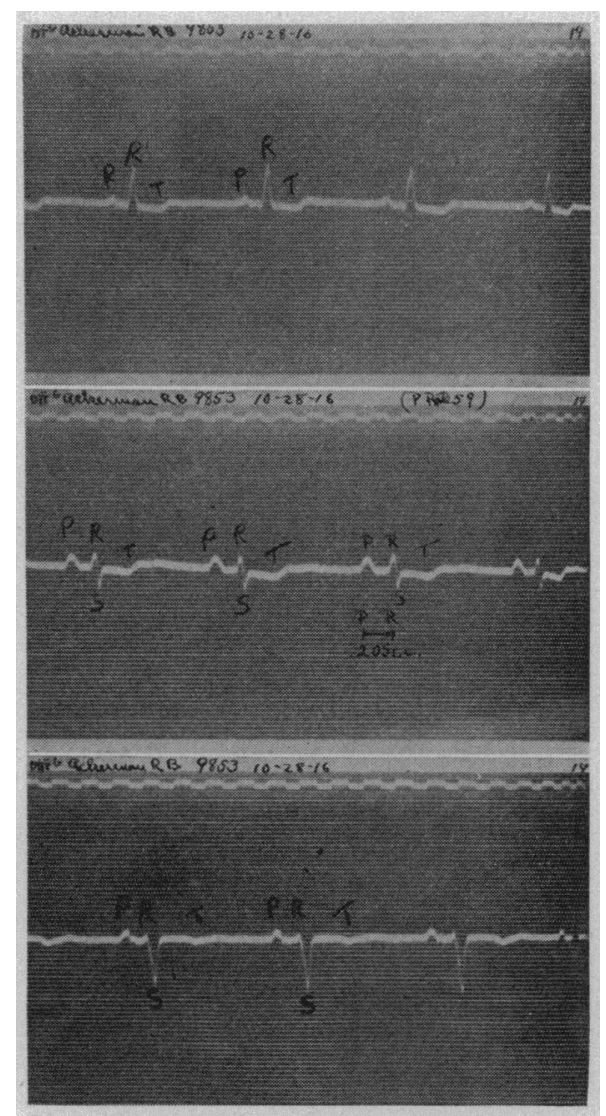

Fig. 23.-(Case 12.) Nov. 28, 1916, thirty-six hours after beginning Eggleston doses. $\mathrm{P}-\mathrm{R}=0.2$ second. T-wave inverted in $\mathrm{D} I$ and $\mathrm{D}$ II, iso-electric, then slight inversion in D III. Pulse rate 59.

An occasional temperature rise to $99.4 \mathrm{~F}$. and once to $100 \mathrm{~F}$. gave rise to a suspicion of mild endocardial infection. Repeated blood cultures showed no growth. Average pulse rate was not decreased under digitalis, although the area of precordial dulness narrowed about $1 \mathrm{~cm}$. Irregularity of pulse from "missed beats" (?) was noted November 17, 1916. The patient was nauseated and vomited once November 18 while taking the official tincture (purpurea). 
Digitalis caused not only a flattening, and later, inversion of T-waves, but also a gradual lengthening of $\mathrm{P}-\mathrm{R}$ interval, this continuing to increase for at least seven days after suspension of the drug. No blocking could be demonstrated then or later.

CASE 12.--R. B. A., man, aged 59 (Hosp. No. 9853), was admitted Oct. 25. 1916; discharged Nov. 18, 1916.

Clinical diagnoses: (1) chronic diffuse nephritis; (2) arteriosclerosis with hypertension (185 systolic, 113 diastolic); (3) hypertrophy, dilatation and decompensation of heart; (4) anasarca; (5) pyorrhea alveolaris and dental caries.

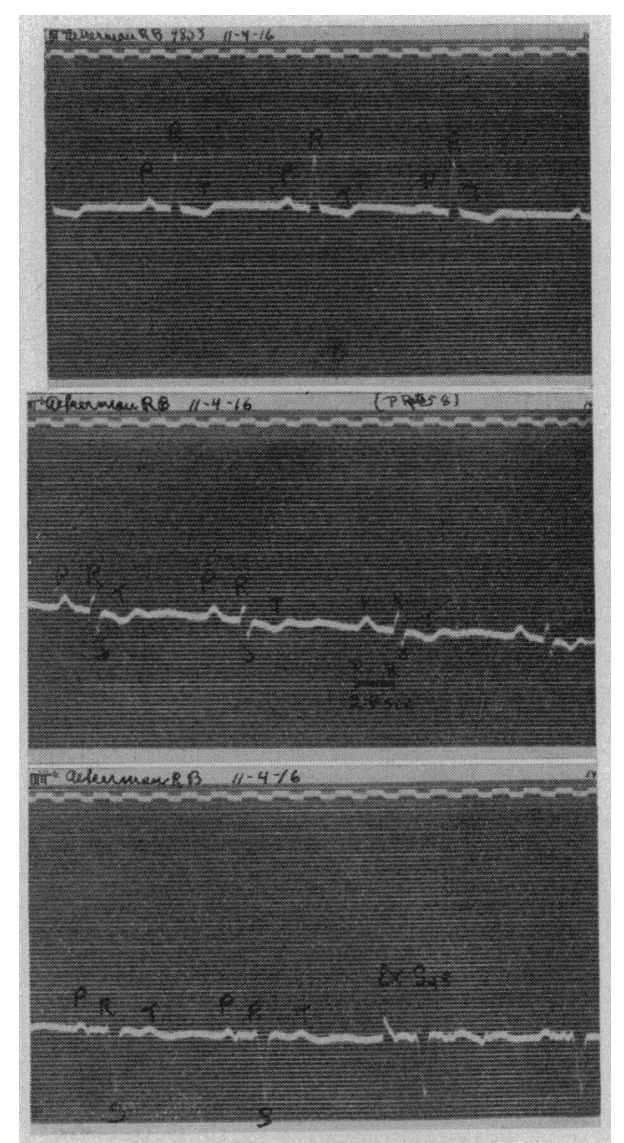

Fig. 24.--(Case 12.) Nov. 4, 1916 (nine days after last Eggleston dose, but after 3 c.c. of tincture on previous day) $P-R=0.24$ second. T-wave shows more inversion in D J ancl D II; nearly iso-clectric and diphasic in D III. Pulse rate 58 .

Weight 185 pounds. The patient was given digitalis as follows:

Infusion digitalis, 180 c.c. Oct. 26 and Oct. 27, 1916, in eighteen hours (Eggleston method); Nov. 3 to Nov. 18,1916 , tincture digitalis. 3 c.c. daily.

Oct. 26, 1916. Electrocardiogram (Fig. 22). P-R $=0.18$ second. $T$ wave slightly inverted in D I; iso-electric in early portion, then slightly positive in D II and D III. Extrasystoles frequent. Pulse rate 75 . 
()et. 28. 1916. Electrocardi gram (Fig. 23). P-R $=0.2$ second. T-wave inverted in D I and D II; iso-electric, then slight inversion in D IIf. No extrasystoles. Pulse rate 59.

Nov. 4. 1916. Electrocardiogram (Fig. 24). P-R $=0.24$ second. T-ware shows mote inversion in D I and D II; nearly iso-electric and diphasic in D ITI. Pulse rute 58 .

Nov. 17. 1916. Electrocardiogram (Fig. 25). P-R $=0.22$ second. T-wave shows still more inversion in D I and D II, with small positive wave in D IIT. Pulse rate 5 S

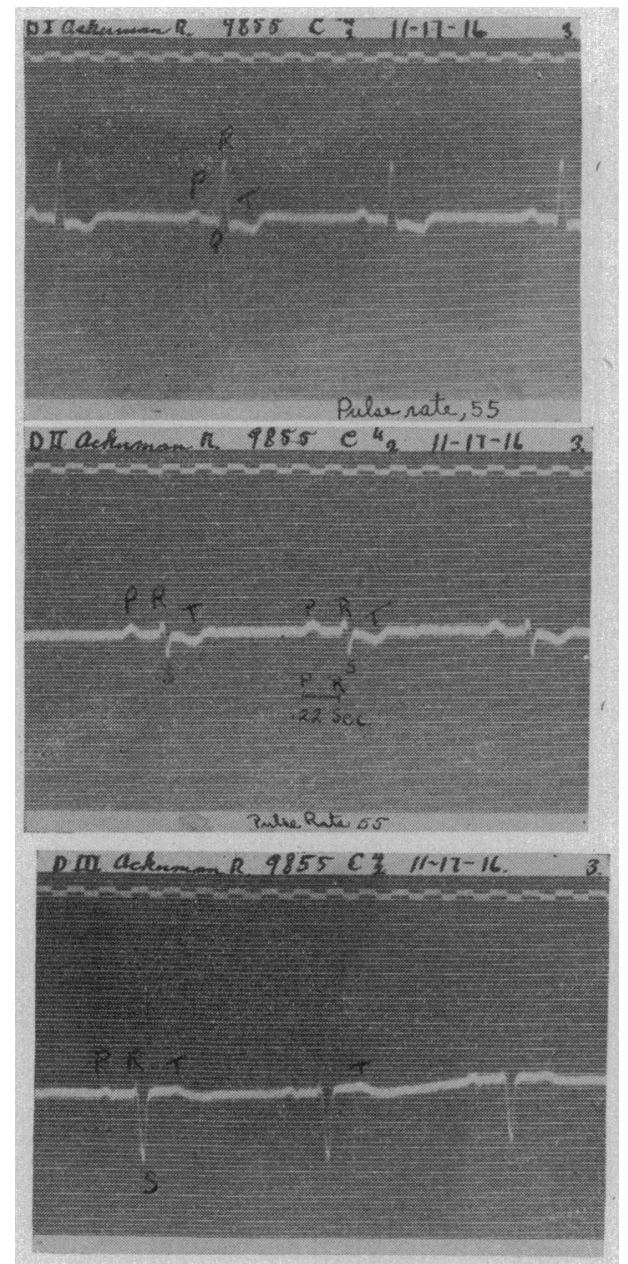

Fig. 25.-(Case 12.) Nov. 17, 1916 (after following Eggleston method with Tr. digitalis, 3 c.c. daily for fourteen days) $P-R=0.22$ second. T-wave shows still more inversion in D I and D II with small positive wave in D IJI. Pulse rate 55 .

A graduai lessening of rate from a normal rate of 75 to one of 55 , three weeks later, occurred. A gradual lengthening of P-R interval from 0.18 second before digitalis, to 0.24 second ten days after begin- 
ning the drug, shows a definite depression of conductivity in the junctional tissues. This is evident within thirty-six hours after beginning the Eggleston doses, but never reaches a high grade, and lessens somewhat even when 3 c.c. of tincture is being given daily. (Compare Figs. 24 and 25.) Block did not occur. Typical changes occur in the T-wave, evident also within thirty-six hours (Fig. 23) and increasing during the administration of the small "tonic" doses. The curves before beginning digitalis (Fig. 22) suggest that the drug had been

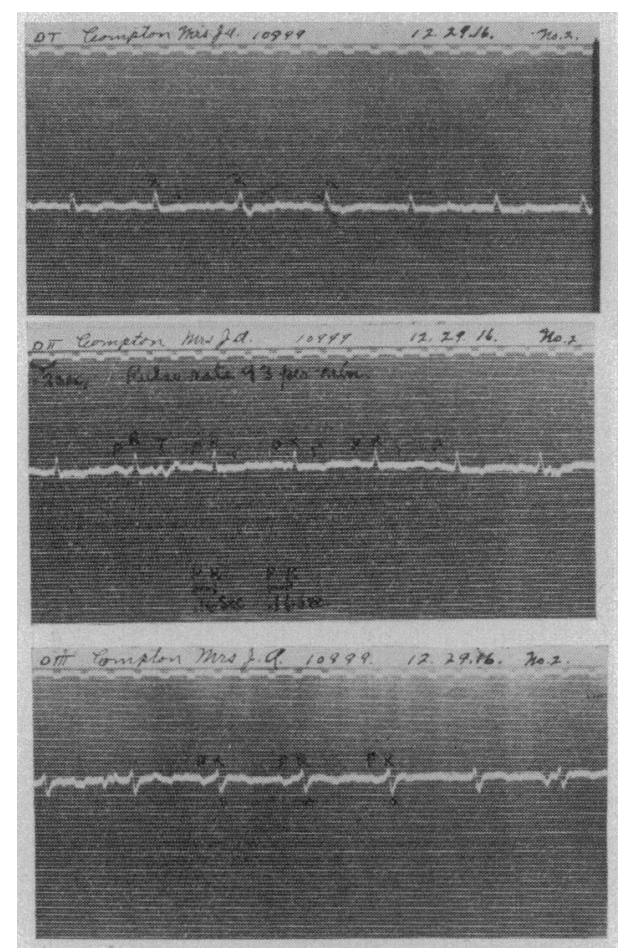

Fig. 26.-(Case 13) Dec. 29, 1916. P-R $=0.16$ second. $\mathrm{T}$ is iso-electric or slightly inverted in all derivations. Pulse rate 93.

taken recently, and this suggestion is heightened by the presence of numerous extrasystoles. The extrasystoles disappeared, however, when the drug was pushed, and other signs of digitalis action appeared.

Throughout this study, this patient improved greatly, and the case illustrates the possibility of definite control with avoidance of undesirable effects when careful and frequent graphic studies are made.

Case 13.-Mrs. J. A. C., aged 58 (Hosp. No. 10299), was admitted Dec. 26, 1916; discharged Feb. 6, 1917.

Clinical diagnoses: (1) pleurisy with effusion, left; (2) displacement of heart to right; (3) gingivitis. 
Weight 125 pounds. The patient was given infusion Digitalis lutea, 120 c.c. in eighteen hours, Dec. 31, 1916, and Jan. 1, 1917; then tincture Digitalis lutea, 4 c.c. daily, Jan. 3, 1917, to Jan. 8, 1917.

Dec. 29, 1916. Electrocardiogram (Fig. 26). $P-R=0.16$ second. $T$ isoelectric or with possibly slight inversion in all derivations. Pulse rate 93 .

Jan. 6. 1917. Electrocardiogram (Fig. 27). Partial block; T-wave distinctly inverted in D I and D II; no distinct change in D III. Pulse rate 62.

Jan. 9, 1917. Electrocardiogram (Fig. 28). Partial block continues, with T-waves showing no change from Jan. 6, 1917 (Fig. 27). Pulse rate 60. Immediately after taking this tracing the patient was given atropin sulphate $0.0017 \mathrm{gm}$. (See Fig. 29 taken twenty-four minutes later.)

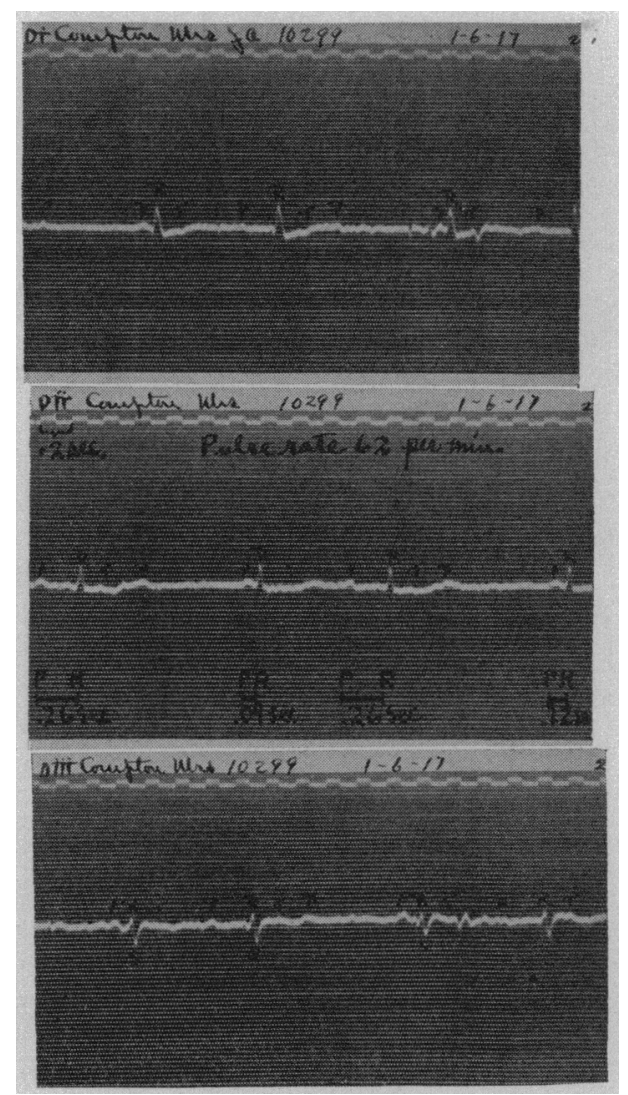

Fig. 27.-(Case 13.) Jan. 6, 1917, partial block. T-wave distinctly inverted in D I and D II; no distinct change in D III. Pulse rate 62.

Jan. 9, 1917, 9:47 to 9:49 a. m. Electrocardiogram (Fig. 29) (taken twentyfour to twenty-six minutes after atropin sulphate $0.0017 \mathrm{gm}$. hypodermically). $\mathrm{P}-\mathrm{R}=0.21$ second. Partial block has disappeared. T-wave shows marked inversion in D I and D II; D III shows first portion of $\mathrm{T}$-wave more strongly positive than in previous tracings. Pulse rate 123 .

The clinical chart shows that this rapid, regular rate gradually disappeared, and slow rate with partial block reappeared in six hours. (Digitalis had been continued for two days after appearance of partial block (Jan. 6, 1917) through an error, as the drug was ordered discontinued at once on recognizing the block.) 
Jan. 11, 1917. Electrocardiogram, shows continuance of partial block, inversion of $\mathrm{T}$-wave, and an occasional ventricular extrasystole, three days after last digitalis and two days after use of atropin.

Jan. 27, 1917. Electrocardiogram. P-R $=0.14$ to 0.17 second, somewhat irregular in length, but no block. T-wave still shows some inversion in D I and D II. D III cannot be distinguished from same derivation before taking digitalis.

Jan. 30, 1917. Electrocardiogram. P-R $=0.16$ second. D I, D II and D III cannot be distinguished from same derivations before taking digitalis.

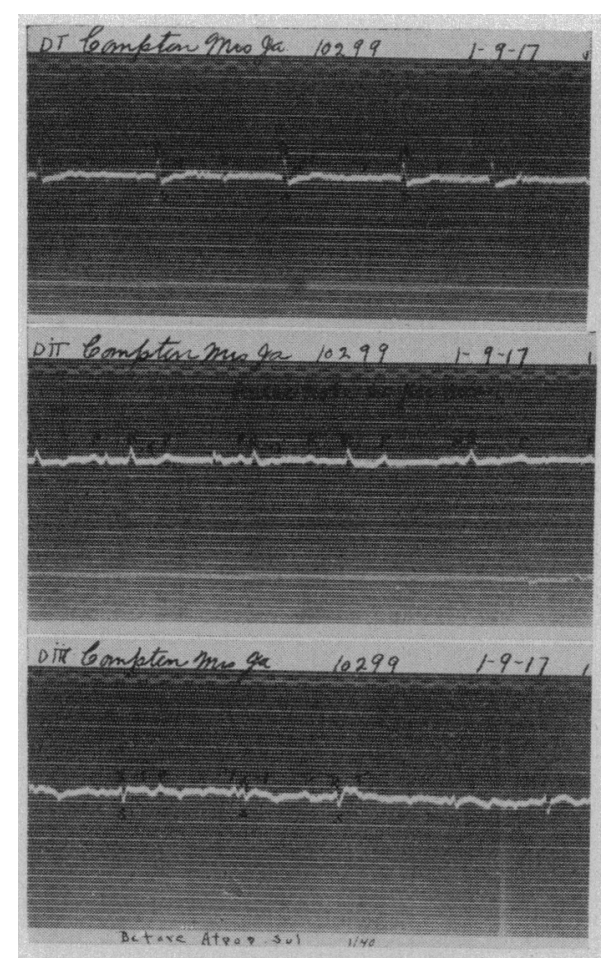

Fig. 28.-(Case 13.) Jan. 9, 1917, partial block continues. T-waves as in Figure 27. Pulse rate 60 .

After taking the Eggleston doses there was no change in rate (104 to 116 per minute), but after beginning "tonic" doses the pulse gradually slowed to a minimum of 84, Jan. 5, 1917. Jan. 6, 1917 the pulse suddenly dropped to 60, with irregularity noted by the nurse. (See also Fig. 27.) Atropin sulphate Jan. 9, 1917 (Fig. 29) removed the block, but caused no change in T-wave.

There was no discomfort, and no untoward symptom and it was not thought necessary to continue atropin. A digitalis effect on the $T$-wave is noted nineteen days after discontinuing the drug.

Case 14.-B. H. C., man, aged 53 (Hosp. No. 9860), was admitted Oct. 26, 1916; discharged Dec. 8, 1916.

Clinical diagnoses: (1) chronic myocarditis; (2) paralysis agitans; (3) pyorrhea alveolaris. 
Ophthalmoscopic examination showed some sclerosis of the retinal vessels.

Weight 161 pounds. The patient was given infusion Digitalis lutea Nov. 5, 1916, and Nov. 6, 1916 (160 c.c. in eighteen hours, "Eggleston dose"). Nov. 7, 1916 , to Nov. 26, 1916, tincture digitalis 3 c.c. daily.

Nov. 4, 1916. Electrocardiogram (Fig. 30). P-R $=0.18$ second. T $4.5 \mathrm{~mm}$. positive in D I; $4 \mathrm{~mm}$. positive in D II; slightly negative, then positive in D III. Ventricular extrasystole in D III. Pulse rate average 96.

Nov. 10, 1916. Electrocardiogram (Fig. 31). $P-Q=0.21$ second. $T$ negative, then $1 \mathrm{~mm}$. positive in D I; $3 \mathrm{~mm}$. negative, then slight positive in D II; $3 \mathrm{~mm}$. negative in D III. Pulse rate, average 96.

Nov. 25, 1916. Electrocardiogram (Fig. 32). Complete block. Increased negativity of $\mathrm{T}$-wave in all leads. Pulse rate, average 55 .

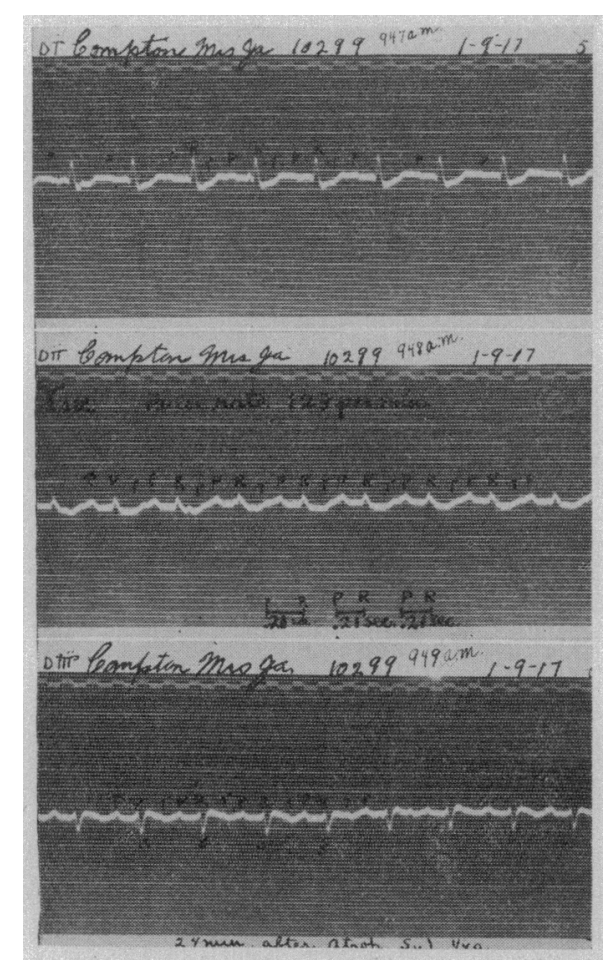

Fig. 29.-(Case 13.) Jan. 9, 1917 (twenty-four to twenty-six minutes after atropin sulphate $0.0017 \mathrm{gm}$. hypodermically) $\mathrm{P}-\mathrm{R}=0.21$ second. Partial block has disappeared, T-wave marked inversion in D I and D II ; first portion more strongly positive than in previous tracings in D III. Pulse rate 123.

Nov. 26, 1916. Electrocardiogram. Complete block continues. Increasing negativity of $\mathrm{T}$-wave. Pulse rate, average 55 .

Nov. 27, 1916. Electrocardiogram. Complete block continues, as does negativity of $\mathrm{T}$-wave. All these phenomena lasted for ten days after suspension of digitalis, then disappeared gradually.

Beginning with a normal (0.18 second) P-R interval, gradual lengthening of this interval occurred, with finally complete auriculoventricular dissociation, as shown in Figure 32, in which in D II the 
ventricular rate is slightly lower than half the auricular rate, the alternate $P$-wave gradually merging in front of the R-wave. In D III the ventricular rate is slightly faster than half the auricular, the alternate I' gradually merging into $R$. Inversion of the T-ware after digitalis occurs als in other cases.

This is the only case of complete block from digitalis in our records. It is of peculiar interest from the fact that the normal P-R interval

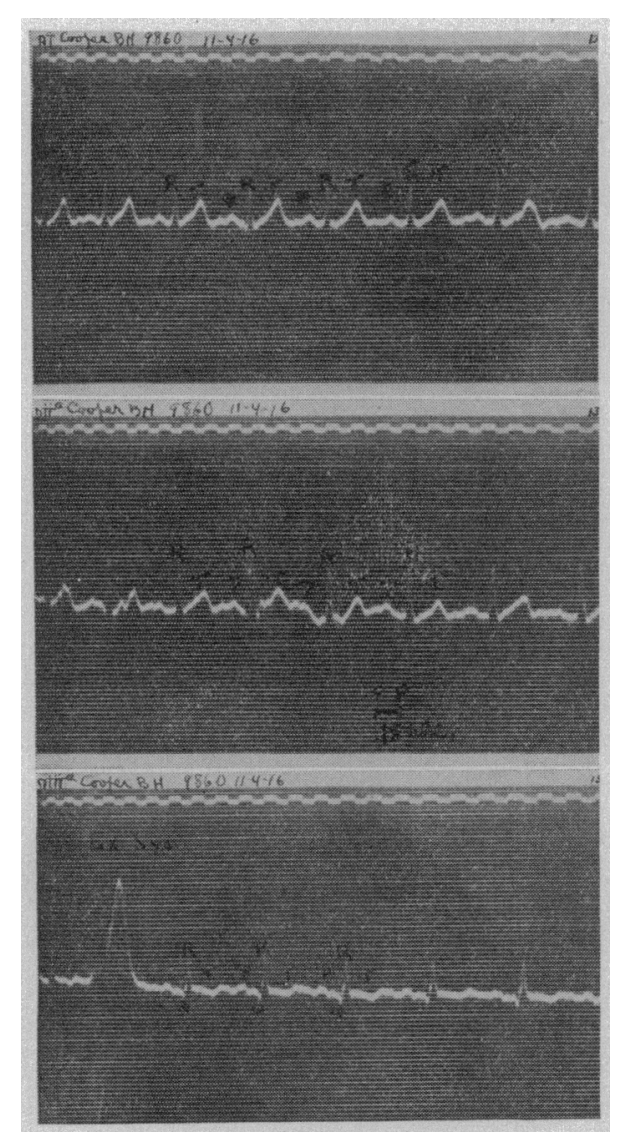

Fig. 30.-(Case 14.) Nov. 4, 1916 (before digitalis) $P-R=0.18$ second. T-wave positive, and high, in D I and D II; slightly negative and then positive in D IIl. Pulse rate averages 96.

before giving digitalis gave no hint of impaired conduction through the bundle.

It the time of admission of this patient, and before giving Digitalis lutea, an occasional extrasystole was noted. There was no increase in the frequency of extrasystoles, even though the block developed, with its consequent slower rate and greater opportunity for the occurrence of ventricular extrasystoles. 
CASe 15.-E. U., man, aged 19 (Hosp. No. 7766).

Clinical diagnoses: (1) chronic valvular disease of heart, mitral and aortic insufficiency and decompensation; (2) chronic passive congestion of liver; (3) ascites; (4) edema legs; (5) dental caries.

Weight 170 pounds. The patient was given tincture digitalis 3 c.c. daily from March 14, 1916, to March 31, 1916, then none until April 19, 1916, when 165 c.c. infusion digitalis, "Eggleston method," were given in eighteen hours.

March 8, 1916. Electrocardiogram. P-R $=0.2$ second. T is a normal upward wave in D I, D II and D III.

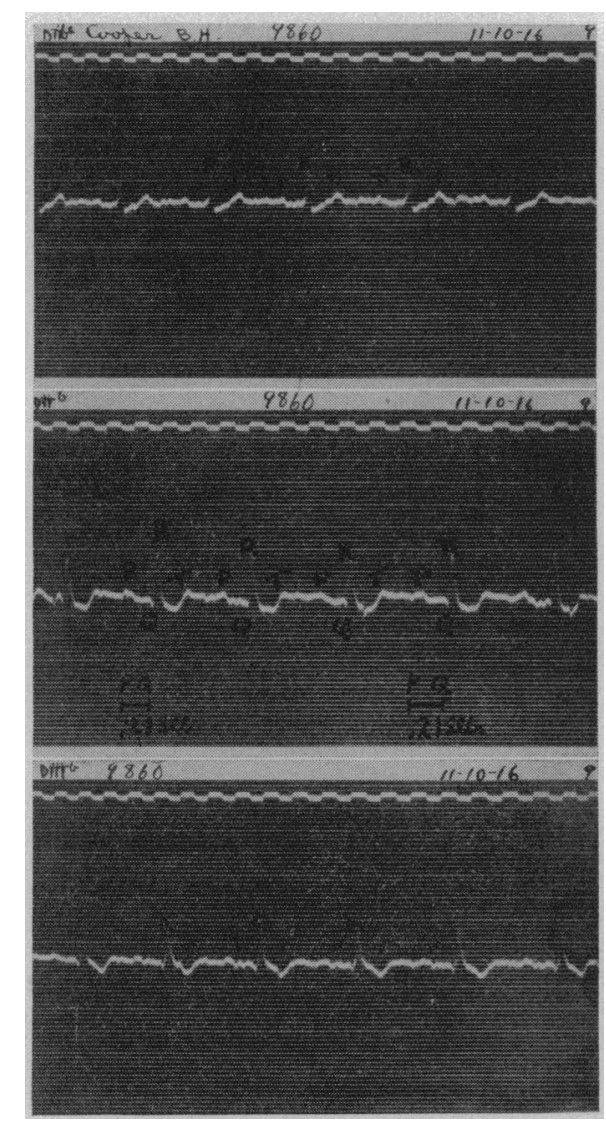

Fig. 31.-(Case 14.) Nov. 10, 1916 (Eggleston dose five days before and 3 c.c. tincture digitalis for three days just preceding this date) $\mathrm{P}-\mathrm{R}=0.21$ second. $\mathrm{T}$ shows marked inversion in all three leads, especially D II. Pulse rate average 96.

April 12, 1916. Electrocardiogram (has had 3 c.c. daily tincture digitalis for eighteen days; suspended twelve days before tracing taken). $P-R=0.22$ second, except where an extrasystole has preceded, when it is 0.01 to 0.02 second longer. T-waves of normal ventricular complexes like those of March 8, 1916, except for moderate flattening in D I. Extrasystoles have been occurring before administration of digitalis. 
April 19, 1916. Electrocardiogram (no digitalis for nineteen days). Taken during attack of acute tonsillitis with temperature $104 \mathrm{~F}$., with a very rapid and regular rate of 146 per minute. $\mathrm{P}-\mathrm{R}=0.25$ second. $\mathrm{P}$ superposed on $\mathrm{T}$.

April 20, 1916. Electrocardiogram (twenty hours after beginning Eggleston (losage). $\mathrm{P}-\mathrm{R}=0.24$. T-wave shows distinct flattening in D II and D III as compared with April 12.1916.

April 26. 1916.-Electrocardiogram. Partial block. (This has been occurring for three days, having hegun three days after the Eggleston doses.) $\mathrm{T}$ is a

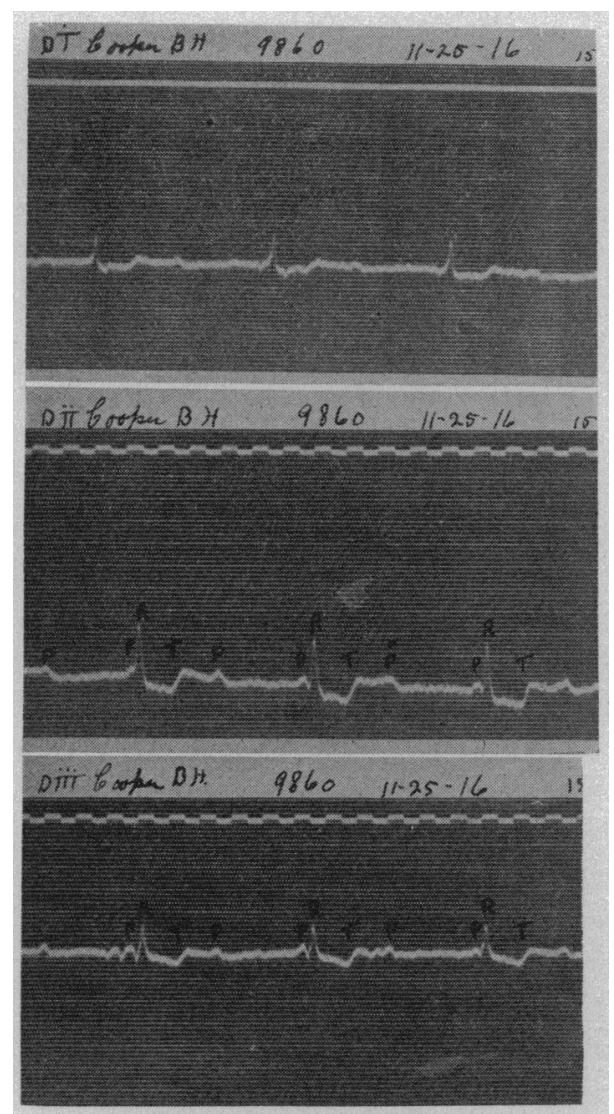

Fig. 32.-(Case 14.) Nov. 25, 1916. Complete block. Increasing negativity of $\mathrm{T}$-wave in all leads. Pulse rate average 55.

higher wave, more like that of March 8, 1916, than of April 20, 1916, after Eggleston doses.

April 28, 1916. Electrocardiogram. Partial block has disappeared. P-R $=$ 0.25 second. There is a marked return of the form of $T$-waves toward that seen before digitalis. Pulse rate 90 . Ventricular extrasystoles occur.

In this case, after having taken "tonic" doses of digitalis, an acute infection occurred with rapid pulse rate. Digitalis in Eggleston doses, given at this time, caused, three days later, a partial block which lasted four days. 
The characteristic changes in $\mathrm{T}$-waves occurred, and are shown at least twenty hours after beginning the drug. The occurrence of extrasystoles was not due to the drug, but was distinctly lessened while the heart was definitely under its influence.

\section{SUMMARY AND CONCLUSIONS}

Our impression is that the Eggleston method is a valuable addition in digitalis therapy, that it gives confidence in the use of the drug, and that the shorter time necessary for securing digitalis effects should give the method wide use.

The method must be used with care to select cases in which these effects are desired. Cases of acute or chronic infections, with the probability of the presence of endocardial infections, should be given the method-if at all-only after careful study, because of the possibility of embolism and, quite as important, in our opinion, the possibility of associated myocardial changes predisposing to block.

The method requires careful study of the patient before, during, and after its administration, and, since it produces powerful and clearcut effect, should be used with extreme care and judgment.

The graphic methods of study show that when given in large enough doses, as by the Eggleston method, digitalis effects may be secured in from sixteen to twenty-four hours.

The electrocardiogram provides a means of accurate study of these effects, showing alterations in the $\mathrm{T}$-wave in all cases studied and also alterations-principally an increase-in conduction time in many.

Alterations in outline of the T-wave accompany physiologic effects of digitalis, and such alterations may persist for many days after withdrawing the drug. In Case 13, persistence of altered $\mathrm{T}$ wave is shown for 19 days; in Case 2, 20 days. In Cases 7, 11, 10 and 2 alteration was found for $6,8,13$, and 14 days, respectively. In our other studies of digitalis action we find it not uncommon for such alterations to persist for 10 days to 2 weeks, the T-wave gradually returning to its normal outline.

The Eggleston method, in cases of decompensation with auricular fibrillation, gives strikingly favorable results. Digitalis effects are shown in from twelve to twenty-four hours in four of our cases (Cases 1, 2, 4 and 5). This is much earlier than results obtained by administering digitalis by older methods, and may therefore be responsible for saving life and is of great clinical significance. In one case (Case 3) electrocardiographic records were not secured until thirtysix hours had elapsed, but a marked effect is shown at this time, and in this case a tremendous urinary output began within twenty-four hours. 
We have not seen the Eggleston doses result either in extrasystoles or in heart block, except in cases giving presumptive evidence of previous digitalis administration. (Case 3 showed paired beats; Case 10, extrasystoles irregularly placed; and Case 15 showed partial heart block of short duration.) In one case (Case 12) prolongation of conduction time from 0.18 to 0.24 second occurred.

The Eggleston method, with 3 or 4 c.c. or more of the tincture daily for several days, has resulted, once in partial and once in complete heart block (Cases 13 and 14). In several instances there has been lengthening of the P-R interval without this becoming so great as to cause block. In these instances no unfavorable effects were noted, and careful watching, with study by graphic methods, has allowed control with prevention of the more severe manifestations of toxic action.

The number of cases in our series is too small to be conclusive as to the time of occurrence of the various effects of digitalis, but in general it appears that in addition to the moderate degree of slowing of the pulse rate, due to a vagus effect on the sino-auricular node, three distinct effects of digitalis may be noted:

(a). Inversion of the $T$-wave, due probably to some effect on conduction through either the finer ramifications of the system of Purkinje fibers, or the musculature itself, or both. This effect is constant in occurrence and accompanies both the desirable and undesirable effects of digitalis, increasing in degree with increase in the amount of the drug given and persisting for several days, often as much as two weeks, and occasionally nearly three weeks, after withdrawal of the drug. This effect is the first to appear and the last to disappear.

(b). Delay in conduction through the bundle of His. The desirability of this effect varies with the conditions in the heart. In auricular fibrillation it is probably largely responsible for the brilliant results so often secured in decompensation with rapid, irregular heart action. In this condition digitalis should be given boldly and with confidence, and the Eggleston method gives most satisfactory results.

In individuals without auricular fibrillation but with a presumably normal conducting tissue, digitalis may be given by the Eggleston method apparently without harm, but no such striking improvement is seen as in cases with auricular fibrillation.

In those patients giving evidence of impaired conduction, as shown by a P-R interval of 0.2 second or over, digitalis must be given with great caution, if at all, and then only in the presence of clear indications for the use of the drug. Any evidence of partial block contraindicates its use or further administration.

The effect on the conducting tissues appears later and after larger 
doses than the effect on the T-wave, except possibly in cases where some impairment of the conducting tissues already exists. It is probable that auricular fibrillation causes some impairment of conduction, or at least renders the conducting tissues sensitive to digitalis, the great frequency of conducted impulses causing a certain degree of exhaustion of the tissue. This impairment may explain in part the prompt, favorable effects of the drug in this condition.

(c). Extrasystoles, ventricular in origin, due to increased irritability of the heart muscle. This effect is undesirable under any circumstances and appears usually last of the three noted, except where the conducting tissues are presumably healthy and not overworked, in which case extrasystoles as a result of digitalis action may occur before changes in conduction time are seen.

Digitalis lutea, the Minnesota first-year leaf, produces effects apparently identical with those of the official Digitalis purpurea, except, possibly, so far as nausea and vomiting are concerned. We have seen these phenomena in man only once after the use of very heavy doses of lutea and are encouraged in studying this drug further with the hope of finding a preparation with digitalis action that produces less of these annoying effects.

We suggest the further trial of Digitalis lutea, and recommend that it be standardized on the basis of "cat unit" rather than on the basis of content of digitalis leaves. In this way a preparation would be available with known physiologic effect, and dosage could be calculated on the basis of body weight. 